\title{
Existence of Nash Equilibrium Points for Markovian Nonzero-sum Stochastic Differential Games with Unbounded Coefficients
}

\author{
Said Hamadène ${ }^{1}$, Rui $\mathrm{Mu}^{12}$ \\ ${ }^{1}$ Université du Maine, LMM, Avenue Olivier Messiaen, 72085 Le Mans, Cedex 9, France \\ ${ }^{2}$ School of Mathematics, Shandong University, Jinan 250100, China \\ E-mails: hamadene@univ-lemans.fr; rui.mu.sdu@gmail.com
}

August 6, 2014

\begin{abstract}
This paper is related to nonzero-sum stochastic differential games in the Markovian framework. We show existence of a Nash equilibrium point for the game when the drift is no longer bounded and only satisfies a linear growth condition. The main tool is the notion of backward stochastic differential equations which, in our case, are multidimensional with continuous coefficient and stochastic linear growth.
\end{abstract}

Keywords: Nonzero-sum Stochastic Differential Games; Nash equilibrium point; Backward Stochastic Differential Equations.

AMS subject classification: 49N70 ; 49N90 ; 91A15.

\section{Introduction}

In this work, we analyze a nonzero-sum stochastic differential game (NZSDG for short) which is described as follows. Assume one has $N$ players $\pi_{1}, \ldots, \pi_{N}$ which intervene on (or control) a system. Each one with the help of an admissible control which is an adapted stochastic process $u^{i}:=\left(u_{t}^{i}\right)_{t \leq T}$ for $\pi_{i}, i=1, \ldots, N$. When the $N$ players make use of a strategy $u:=\left(u^{1}, \ldots, u^{N}\right)$, the dynamics of the controlled system is a process $\left(x_{t}^{u}\right)_{t \leq T}$ solution of the following standard stochastic differential equation (SDE for short):

$$
d x_{t}^{u}=f\left(t, x_{t}^{u}, u_{t}^{1}, \ldots, u_{t}^{N}\right) d t+\sigma\left(t, x_{t}^{u}\right) d B_{t} \text { for } t \leq T \text { and } x_{0}=x ;
$$

$B:=\left(B_{t}\right)_{t \leq T}$ is a Brownian motion. The control actions are not free and generate for each player $\pi_{i}, i=1, \ldots, N$, a payoff which amounts to

$$
J_{i}\left(u^{1}, \ldots, u^{N}\right):=\mathbf{E}\left[g^{i}\left(x_{T}^{u}\right)+\int_{0}^{T} h_{i}\left(s, x_{s}^{u}, u_{s}\right) d s\right] .
$$

A Nash equilibrium point (NEP for short) for the players is a strategy $u^{*}:=\left(u^{1, *}, \ldots, u^{N, *}\right)$ of control of the system which has the feature that each player $\pi_{i}$ who takes unilaterally the decision to deviate from $u^{i, *}$, is penalized: For all $i=1, \ldots, N$, for all control $u^{i}$ of player $\pi_{i}$,

$$
J_{i}\left(u^{*}\right) \leq J_{i}\left(\left[u^{*,-i} \mid u^{i}\right]\right)
$$

where $\left[u^{*,-i} \mid u^{i}\right]:=\left(u^{1, *}, \ldots, u^{i-1, *}, u^{i}, u^{i+1, *}, \ldots, u^{N, *}\right)$.

In the case when $N=2$ and $J_{1}+J_{2}=0$, this game reduces to the well known zero-sum differential game which is well documented in several works and from several points of view (see e.g. [2], [3], [5], [6], [9], [10], [14], [15], [20] etc. and the references inside). 
Comparatively, the nonzero-sum differential game is so far less considered even though there are some works on the subject, including [4], [11], [16], [13], [17], [18], [22], [23], [24], [28], etc.). In these works, the objectives are various and so are the approaches, usually based on partial differential equations (PDEs) $([11,24])$ or backward SDEs $([16,17,18,23,22])$. On the other hand, it should be pointed out that the frameworks in those papers are not the same. Some of them consider strategies as control actions for the players (e.g. [4], [23], [28]) while others deal with the control against control setting (e.g. [13], [16, 17, 18]). The first ones, formulated usually in the framework of two players, allow to study the case where the diffusion coefficient $\sigma$ is controlled. In the latter ones, $\sigma$ does not depend on the controls. However those papers do not reach the same objective. Note that for the control against control zero-sum game, Pham and Zhang [26] and M.Sirbu [29] have overcome this restriction related to the independence of $\sigma$ on the controls.

In the present article, we study the nonzero-sum game of type control against control with the diffusion process $\sigma$ independent of controls, in the same line as in the paper by Hamadène et al. [16]. But in [16], the setting concerns only the case when the coefficients $f$ and $\sigma$ of the diffusion (1.1) are bounded. According to our knowledge the setting where those coefficients are not bounded and of linear growth is not considered yet. Therefore the main objective of this work is to relax as much as possible the boundedness of the coefficients $f$ (mainly) and $\sigma$ (which is not bounded as stated in the final extension). The novelty of the paper is that we show the existence of a Nash equilibrium point for the NZSDG when $f$ is no longer bounded but only satisfies the linear growth condition. As in [16] our approach is based on backward SDEs and basically the problem turns into studying its associated multi-dimensional BSDE which is of linear growth $\omega$ by $\omega$. Under the generalized Isaacs hypothesis and the domination condition of laws of solutions of (1.1), which is satisfied when Hörmander's condition on $\sigma$ is satisfied, we show that the latter BSDE has a solution which then provides a NEP for the NZSDG.

The paper is organized as follows:

In Section 2 we fix the setting of the problem and recall some results which play an important role in our study. The formulation we adopt is of weak type. On the other hand for the sake of simplicity we have made the presentation for $N=2$. The generalization to the situation where $N \geq 3$ is formal and can be carried out in the same spirit. Section 3 is devoted to the link between the game and BSDEs. We first express the payoffs of the game in using solutions of BSDEs whose integrability is not standard. Then we show that the existence of a NEP for the game turns into the existence of a solution of a specific BSDE which is of multi-dimensional type and linear growth $\omega$ by $\omega$. It plays a role of a verification theorem for the NZSDG. In Section 4 we show that this specific BSDE has a solution when the generalized Isaacs condition is fulfilled and the laws of the dynamics of the non-controlled system satisfy the so-called $L^{q}$ domination condition. This latter is especially satisfied when the diffusion coefficient $\sigma$ satisfies the well known Hörmander's condition. Our method is based on: (i) the introduction of an approximating scheme of BSDEs which is well-posed since the coefficients are Lipschitz. In this markovian framework of randomness, the solutions $\left(Y^{n}, Z^{n}\right), n \geq 1$, of this scheme can be represented via deterministic functions $\left(\varpi^{n}, v^{n}\right), n \geq 1$, and the Markov process as well ; (ii) sharp estimates for $\left(Y^{n}, Z^{n}\right)$ and $\left(\varpi^{n}, v^{n}\right)$ and the $L^{q}$-domination condition enable us to obtain the strong convergence of a subsequence $\left(\varpi_{n_{k}}\right)_{k \geq 1}$ from a weak convergence in an appropriate space. This yields the strong convergence of the corresponding subsequences $\left(Y^{n_{k}}\right)_{k \geq 1}$ and $\left(Z^{n_{k}}\right)_{k \geq 1}$; (iii) we finally show that the limit of $\left(Y^{n_{k}}, Z^{n_{k}}\right)_{k \geq 1}$ is a solution for the BSDE associated with the NZSDG. At the end of this section we provide an example which illustrates our result. We also discuss possible extensions of our findings to the case when both the drift $f$ and diffusion coefficient $\sigma$ of (1.1) are not bounded. 


\section{Setting of the problem}

Let $T>0$ and let $(\Omega, \mathcal{F}, \mathbf{P})$ be a probability space on which is defined an $m$-dimensional Brownian motion $B:=\left(B_{t}\right)_{0 \leq t \leq T}$. For $t \leq T$, let us denote by $\left(F_{t}:=\sigma\left(B_{u}, u \leq t\right)\right)_{t \leq T}$ the natural filtration of $B$ and $\left(\mathcal{F}_{t}\right)_{t \leq T}$ the completion of $\left(F_{t}\right)_{t \leq T}$ with the $\mathbf{P}$-null sets of $\mathcal{F}$, which then satisfies the usual conditions. Let $\mathcal{P}$ be the $\sigma$-algebra on $[0, T] \times \Omega$ of $\mathcal{F}_{t}$-progressively measurable sets. Let $p \geq 1$ be a real constant and $t \in[0, T]$ fixed. We then define the following spaces:

- $L_{T}^{p}\left(\mathbf{R}^{m}\right)=\left\{\xi: \mathcal{F}_{T}\right.$-measurable and $\mathbf{R}^{m}$-valued random variable s.t. $\left.\mathbf{E}\left[|\xi|^{p}\right]<\infty\right\}$;

- $\mathcal{S}_{t, T}^{p}\left(\mathbf{R}^{m}\right)=\left\{\varphi=\left(\varphi_{s}\right)_{t \leq s \leq T}: \mathcal{P}\right.$-measurable and $\mathbf{R}^{m}$-valued s.t. $\left.\mathbf{E}\left[\sup _{t \leq s \leq T}\left|\varphi_{s}\right|^{p}\right]<\infty\right\}$ $; \mathcal{S}_{0, T}^{p}\left(\mathbf{R}^{m}\right)$ is simply denoted by $\mathcal{S}_{T}^{p}\left(\mathbf{R}^{m}\right)$;

- $\mathcal{H}_{t, T}^{p}\left(\mathbf{R}^{m}\right)=\left\{Z=\left(Z_{s}\right)_{t \leq s \leq T}: \mathcal{P}\right.$-measurable and $\mathbf{R}^{m}$-valued s.t. $\mathbf{E}\left[\left(\int_{t}^{T}\left|Z_{s}\right|^{2} d s\right)^{p / 2}\right]<$ $\infty\} ; \mathcal{H}_{0, T}^{p}\left(\mathbf{R}^{m}\right)$ is simply denoted by $\mathcal{H}_{T}^{p}\left(\mathbf{R}^{m}\right)$.

Next let $\sigma$ be a matrix function defined as:

$$
\begin{array}{cl}
\sigma:[0, T] \times \mathbf{R}^{m} & \longrightarrow \mathbf{R}^{m \times m} \\
(t, x) & \longmapsto \sigma(t, x)
\end{array}
$$

and which satisfies the following assumptions:

\section{Assumptions (A1)}

(i) $\sigma$ is uniformly Lipschitz w.r.t $x$. i.e. there exists a constant $C_{1}$ such that,

$$
\forall t \in[0, T], \forall x, x^{\prime} \in \mathbf{R}^{m}, \quad\left|\sigma(t, x)-\sigma\left(t, x^{\prime}\right)\right| \leq C_{1}\left|x-x^{\prime}\right|
$$

(ii) $\sigma$ is invertible and bounded and its inverse is bounded, i.e., there exits a constant $C_{2}$ such that

$$
\forall(t, x) \in[0, T] \times \mathbf{R}^{m}, \quad|\sigma(t, x)|+\left|\sigma^{-1}(t, x)\right| \leq C_{2} .
$$

Remark 2.1. Hörmander's condition.

Under (A1), there exists a real constant $\Upsilon>0$ such that for any $(t, x) \in[0, T] \times \mathbf{R}^{m}$,

$$
\Upsilon . I \leq \sigma(t, x) \cdot \sigma^{\top}(t, x) \leq \Upsilon^{-1} \cdot I
$$

where $I$ is the identity matrix of dimension $m$.

Next let $(t, x) \in[0, T] \times \mathbf{R}^{m}$. Under the Assumptions (A1), (i)-(ii), we know that there exists a process $\left(X_{s}^{t, x}\right)_{s \leq T}$ that satisfies the following stochastic differential equation (see e.g. Karatzas and Shreve, pp.289, 1991 [21]):

$$
X_{s}^{t, x}=x+\int_{t}^{s} \sigma\left(r, X_{r}^{t, x}\right) d B_{r}, \quad \forall s \in[t, T] \text { and } X_{s}^{t, x}=x \text { for } s \in[0, t] .
$$

Hereafter for sake of simplicity we will deal with the setting of two players. However the generalization to the case of $N(\geq 3)$ players is formal and just a question of writing (see the comment of Remark 4.1). Also let us denote by $U_{1}$ and $U_{2}$ two compact metric spaces and let 
$\mathcal{M}_{1}$ (resp. $\mathcal{M}_{2}$ ) be the set of $\mathcal{P}$-measurable processes $u=\left(u_{t}\right)_{t \leq T}$ (resp. $v=\left(v_{t}\right)_{t \leq T}$ ) with values in $U_{1}$ (resp. $\left.U_{2}\right)$. We denote by $\mathcal{M}$ the set $\mathcal{M}_{1} \times \mathcal{M}_{2}$ and call it the set of admissible controls for the players.

Let $f$ be a Borelian function from $[0, T] \times \mathbf{R}^{m} \times U_{1} \times U_{2}$ into $\mathbf{R}^{m}$ and for $i=1,2$ let $h_{i}$ and $g^{i}$ be Borelian functions from $[0, T] \times \mathbf{R}^{m} \times U_{1} \times U_{2}$ (resp. $\left.\mathbf{R}^{m}\right)$ into $\mathbf{R}$ which satisfy:

\section{Assumptions (A2)}

(i) $f$ is of linear growth w.r.t $x$, i.e. there exists a constant $C_{3}$ such that $|f(t, x, u, v)| \leq$ $C_{3}(1+|x|)$, for any $(t, x, u, v) \in[0, T] \times \mathbf{R}^{m} \times U_{1} \times U_{2}$.

(ii) for $i=1,2 h_{i}$ is of polynomial growth w.r.t $x$, i.e., there exists a constant $C_{4}$ and $\gamma \geq 0$ such that $\left|h_{i}(t, x, u, v)\right| \leq C_{4}\left(1+|x|^{\gamma}\right)$ for any $(t, x, u, v) \in[0, T] \times \mathbf{R}^{m} \times U_{1} \times U_{2}$.

(iii) for $i=1,2, g^{i}$ is of polynomial growth with respect to $x$, i.e. there exist constants $C_{5}$ and $\gamma \geq 0$ such that $\left|g^{i}(x)\right| \leq C_{5}\left(1+|x|^{\gamma}\right), \forall x \in \mathbf{R}^{m}$. follows:

For $(u, v) \in \mathcal{M}$, let $\mathbf{P}_{(t, x)}^{(u, v)}$ be the measure on $(\Omega, \mathcal{F})$ whose density function is defined as

$$
\frac{d \mathbf{P}_{(t, x)}^{(u, v)}}{d \mathbf{P}}=\zeta_{T}\left(\sigma^{-1}\left(\cdot, X_{.}^{t, x}\right) f\left(\cdot, X_{.}^{t, x}, u ., v .\right)\right),
$$

where for any measurable $\mathcal{F}_{t}$-adapted process $\eta:=\left(\eta_{s}\right)_{s \leq T}$ we define,

$$
\zeta_{s}(\eta):=e^{\int_{0}^{s} \eta_{r} d B_{r}-\frac{1}{2} \int_{0}^{s}\left|\eta_{r}\right|^{2} d r}, \forall s \leq T .
$$

Thanks to the Assumptions (A1) and (A2)-(i) on $\sigma$ and $f$, we can infer that $\mathbf{P}_{(t, x)}^{(u, v)}$ is a probability on $(\Omega, \mathcal{F})$ (see Appendix A of N. El-Karoui and S. Hamadène [7] or KaratzasShreve [21], pp.200). Then by Girsanov's theorem (Girsanov, [12]), the process $B^{(u, v)}:=$ $\left(B_{s}-\int_{0}^{s} \sigma^{-1}\left(r, X_{r}^{t, x}\right) f\left(r, X_{r}^{t, x}, u_{r}, v_{r}\right) d r\right)_{s \leq T}$ is a $\left(\mathcal{F}_{s}, \mathbf{P}_{(t, x)}^{(u, v)}\right)$-Brownian motion and $\left(X_{s}^{t, x}\right)_{s \leq T}$ satisfies the following stochastic differential equation,

$$
d X_{s}^{t, x}=f\left(s, X_{s}^{t, x}, u_{s}, v_{s}\right) d s+\sigma\left(s, X_{s}^{t, x}\right) d B_{s}^{(u, v)}, \text { for } s \in[t, T] \text { and } X_{s}^{t, x}=x \text { for } s<t .
$$

In general, the process $\left(X_{s}^{t, x}\right)_{s \leq T}$ is not adapted with respect to the filtration generated by the Brownian motion $\left(B_{s}^{(u, v)}\right)_{s \leq T}$, therefore $\left(X_{s}^{t, x}\right)_{s \leq T}$ is called a weak solution for the SDE (2.4).

Next let us fix $(t, x)$ to $\left(0, x_{0}\right)$ and for $i=1,2$, let us define the payoffs of the players by:

$$
J^{i}(u, v)=\mathbf{E}_{\left(0, x_{0}\right)}^{(u, v)}\left[\int_{0}^{T} h_{i}\left(s, X_{s}^{0, x_{0}}, u_{s}, v_{s}\right) d s+g^{i}\left(X_{T}^{0, x_{0}}\right)\right],
$$

where $\mathbf{E}_{\left(0, x_{0}\right)}^{(u, v)}(\cdot)$ is the expectation under the probability $\mathbf{P}_{\left(0, x_{0}\right)}^{(u, v)}$. Hereafter $\mathbf{E}_{\left(0, x_{0}\right)}^{(u, v)}(\cdot)$ (resp. $\left.\mathbf{P}_{\left(0, x_{0}\right)}^{(u, v)}\right)$ will be simply denoted by $\mathbf{E}^{(u, v)}().\left(\right.$ resp. $\left.\mathbf{P}^{(u, v)}\right)$.

Our problem is to find an admissible control $\left(u^{*}, v^{*}\right)$ such that

$$
J^{1}\left(u^{*}, v^{*}\right) \leq J^{1}\left(u, v^{*}\right) \text { and } J^{2}\left(u^{*}, v^{*}\right) \leq J^{2}\left(u^{*}, v\right) \text { for any }(u, v) \in \mathcal{M} .
$$

The control $\left(u^{*}, v^{*}\right)$ is called a Nash equilibrium point for the nonzero-sum stochastic differential game. 
Next we define the Hamiltonian functions $H_{i}, i=1,2$, of the game from $[0, T] \times \mathbf{R}^{2 m} \times U_{1} \times U_{2}$ into $\mathbf{R}$ by:

$$
H_{i}(t, x, p, u, v)=p \sigma^{-1}(t, x) f(t, x, u, v)+h_{i}(t, x, u, v),
$$

and we introduce the following assumption (A3) called the generalized Isaacs condition.

Assumption (A3): Generalized Isaacs condition.

(i) There exist two Borelian applications $u_{1}^{*}$, $u_{2}^{*}$ defined on $[0, T] \times \mathbf{R}^{3 m}$, with values in $U_{1}$ and $U_{2}$, respectively, such that for any $(t, x, p, q, u, v) \in[0, T] \times \mathbf{R}^{3 m} \times U_{1} \times U_{2}$, we have:

$$
H_{1}^{*}(t, x, p, q)=H_{1}\left(t, x, p, u_{1}^{*}(t, x, p, q), u_{2}^{*}(t, x, p, q)\right) \leq H_{1}\left(t, x, p, u, u_{2}^{*}(t, x, p, q)\right)
$$

and

$$
H_{2}^{*}(t, x, p, q)=H_{2}\left(t, x, q, u_{1}^{*}(t, x, p, q), u_{2}^{*}(t, x, p, q)\right) \leq H_{2}\left(t, x, q, u_{1}^{*}(t, x, p, q), v\right) .
$$

(ii) the mapping $(p, q) \in \mathbf{R}^{2 m} \longmapsto\left(H_{1}^{*}, H_{2}^{*}\right)(t, x, p, q) \in \mathbf{R}$ is continuous for any fixed $(t, x)$.

Remark 2.2. This condition has been already considered by A. Friedman in [10] for the same purpose as ours in this paper. But the treatment of the problem he used is the PDE approach.

In order to show that the game has a Nash equilibrium point, it is enough to show that its associated BSDE, which is multi-dimensional and with a continuous generator (see Theorem 3.1 below) has a solution. Therefore the main objective of the next section is to study the connection between NZSDGs and BSDEs.

\section{Nonzero-sum differential game problem and BSDEs.}

Let $(t, x) \in[0, T] \times \mathbf{R}^{m}$ and $\left(\theta_{s}^{t, x}\right)_{s \leq T}$ be the solution of the following forward stochastic differential equation:

$$
\left\{\begin{aligned}
d \theta_{s} & =b\left(s, \theta_{s}\right) d s+\sigma\left(s, \theta_{s}\right) d B_{s}, & s \in[t, T] ; \\
\theta_{s} & =x, & s \in[0, t]
\end{aligned}\right.
$$

where $\sigma:[0, T] \times \mathbf{R}^{m} \rightarrow \mathbf{R}^{m \times m}$ satisfies the Assumptions (A1) and $b:[0, T] \times \mathbf{R}^{m} \rightarrow \mathbf{R}^{m}$ is a measurable function which satisfies the following assumption:

Assumption (A4): The function $b$ is uniformly Lipschitz w.r.t $x$ and of linear growth, i.e., there exist constants $C_{6}$ and $C_{7}$ such that:

$$
\forall t \in[0, T], \forall x, x^{\prime} \in \mathbf{R}^{m},\left|b(t, x)-b\left(t, x^{\prime}\right)\right| \leq C_{6}\left|x-x^{\prime}\right| \text { and }|b(t, x)| \leq C_{7}(1+|x|) .
$$

It is well-known that, under (A1) and (A4), the stochastic process $\left(\theta_{s}^{t, x}\right)_{s \leq T}$ satisfies the following estimate, see for example (Karatzas, I. 1991 [21] pp.306):

$$
\forall q \in[1, \infty), \quad \mathbf{E}\left[\left(\sup _{s \leq T}\left|\theta_{s}^{t, x}\right|\right)^{2 q}\right] \leq C\left(1+|x|^{2 q}\right) .
$$

As a particular case we have a similar estimate for the process $X^{t, x}$, i.e.,

$$
\forall q \in[1, \infty), \quad \mathbf{E}\left[\left(\sup _{s \leq T}\left|X_{s}^{t, x}\right|\right)^{2 q}\right] \leq C\left(1+|x|^{2 q}\right) .
$$


Finally note that we have also a similar estimate for weak solutions of SDEs of types (2.4), i.e., if $(u, v)$ belongs to $\mathcal{M}$ then

$$
\forall q \in[1, \infty), \quad \mathbf{E}_{(t, x)}^{(u, v)}\left[\left(\sup _{s \leq T}\left|X_{s}^{t, x}\right|\right)^{2 q}\right] \leq C\left(1+|x|^{2 q}\right) .
$$

Next let us recall the following result by U.G.Haussmann [19] related to integrability of the exponential local martingale defined in (2.3).

Lemma 3.1. Assume (A1)-(i),(ii) and (A4) and let $\left(\theta_{s}^{t, x}\right)_{s \leq T}$ be the solution of (3.1). Let $\varphi$ be a $\mathcal{P} \otimes \mathcal{B}\left(\mathbf{R}^{m}\right)$-measurable application from $[0, T] \times \Omega \times \mathbf{R}^{m}$ to $\mathbf{R}^{m}$ which is uniformly of linear growth, that is, $\mathbf{P}$-a.s., $\forall(s, x) \in[0, T] \times \mathbf{R}^{m}$,

$$
|\varphi(s, \omega, x)| \leq C_{8}(1+|x|) .
$$

Then, there exists $p \in(1,2)$ and a constant $C$, where $p$ depends only on $C_{2}, C_{6}, C_{7}, C_{8}, m$ while the constant $C$, depends only on $m$ and $p$, but not on $\varphi$, such that:

$$
\mathbf{E}\left[\left(\zeta_{T}\left(\varphi\left(s, \theta_{s}^{t, x}\right)\right)\right)^{p}\right] \leq C,
$$

where the process $\zeta\left(\varphi\left(s, \theta_{s}^{t, x}\right)\right)$ is the density function defined in (2.3).

As a by-product we have:

Corollary 3.1. Let $(u, v)$ be an admissible control for the players and $(t, x) \in[0, T] \times \mathbf{R}^{m}$. Then there exists $p>1$ such that

$$
\mathbf{E}\left[\left(\zeta_{T}\left(\sigma\left(s, X_{s}^{t, x}\right)^{-1} f\left(s, X_{s}^{t, x}, u_{s}, v_{s}\right)\right)\right)^{p}\right] \leq C .
$$

Next we give a preliminary result which characterizes $J(u, v)$ of $(2.5)$ from its associated BSDE. This result generalizes the one by S. Hamadène, and J. P. Lepeltier, 1995b [15] (Theorem I.3). The main improvement is that the drift of the diffusion, weak solution of (2.4), is not bounded anymore but is instead of linear growth.

Proposition 3.1. Assume that Assumptions (A1), and (A2) on $f, h_{i}, g^{i}, i=1,2$, are fulfilled. Then for any pair $(u, v) \in \mathcal{M}$, there exists a pair of $\mathcal{P}$-measurable processes $\left(W^{i,(u, v)}, Z^{i,(u, v)}\right)$, $i=1,2$, with values in $\mathbf{R} \times \mathbf{R}^{m}$ such that:

(i) For any $q \geq 1$ and $i=1,2$, we have,

$$
\mathbf{E}^{(u, v)}\left[\sup _{0 \leq s \leq T}\left|W_{s}^{i,(u, v)}\right|^{q}+\left(\int_{0}^{T}\left|Z_{s}^{i,(u, v)}\right|^{2} d s\right)^{\frac{q}{2}}\right]<\infty .
$$

(ii) For $t \leq T$,

$$
W_{t}^{i,(u, v)}=g^{i}\left(X_{T}^{0, x_{0}}\right)+\int_{t}^{T} H_{i}\left(s, X_{s}^{0, x_{0}}, Z_{s}^{i,(u, v)}, u_{s}, v_{s}\right) d s-\int_{t}^{T} Z_{s}^{i,(u, v)} d B_{s} .
$$

The solution of BSDE (3.6)-(3.7) is unique, moreover, $W_{0}^{i,(u, v)}=J^{i}(u, v)$ for $i=1,2$. 
Proof. We will give the proof for $i=1$ and of course it is similar for $i=2$. So for $(u, v) \in \mathcal{M}$, the process $\left(X_{s}^{0, x_{0}}\right)_{s \leq T}$ is a weak solution of the following stochastic differential equation:

$$
d X_{s}^{0, x_{0}}=f\left(s, X_{s}^{0, x_{0}}, u_{s}, v_{s}\right) d s+\sigma\left(s, X_{s}^{0, x_{0}}\right) d B_{s}^{(u, v)}, \quad s \leq T \text { and } X_{0}^{0, x_{0}}=x_{0},
$$

where $B^{(u, v)}$ is a Brownian motion under $\mathbf{P}^{(u, v)}$. Let us define the process $\left(W_{t}^{1,(u, v)}\right)_{t \leq T}$ as follows:

$$
\forall t \leq T, W_{t}^{1,(u, v)} \triangleq \mathbf{E}^{(u, v)}\left[g^{1}\left(X_{T}^{0, x_{0}}\right)+\int_{t}^{T} h_{1}\left(s, X_{s}^{0, x_{0}}, u_{s}, v_{s}\right) d s \mid \mathcal{F}_{t}\right] .
$$

Since the functions $g^{1}(x)$ and $h_{1}(s, x, u, v)$ are of polynomial growth w.r.t $x$, we have, for any $r \geq 1$,

$$
\begin{aligned}
& \mathbf{E}^{(u, v)}\left[\left|g^{1}\left(X_{T}^{0, x_{0}}\right)+\int_{0}^{T}\right| h_{1}\left(s, X_{s}^{0, x_{0}}, u_{s}, v_{s}\right)|d s|^{2 r}\right] \\
& \quad \leq \mathbf{E}^{(u, v)}\left[C\left(1+\sup _{s \leq T}\left|X_{s}^{0, x_{0}}\right|^{2 \gamma r}\right)\right] \\
& \quad \leq C\left(1+\left|x_{0}\right|^{2 \gamma r}\right) .
\end{aligned}
$$

The last inequality is due to estimate (3.4). Then (3.9) implies that the process $\left(W_{t}^{1,(u, v)}\right)_{t \leq T}$ is well defined.

Next for notation simplicity for any $t \leq T$, we denote by $\zeta_{t}$ the density function $\zeta_{t}\left(\sigma^{-1}\left(s, X_{s}^{0, x_{0}}\right) f\left(s, X_{s}^{0, x_{0}}, u_{s}, v_{s}\right)\right)$. Therefore,

$$
\begin{aligned}
W_{t}^{1,(u, v)}= & \left(\zeta_{t}\right)^{-1} \mathbf{E}\left[\zeta_{T} \cdot\left(g^{1}\left(X_{T}^{0, x_{0}}\right)+\int_{t}^{T} h_{1}\left(s, X_{s}^{0, x_{0}}, u_{s}, v_{s}\right) d s\right) \mid \mathcal{F}_{t}\right] \\
= & \left(\zeta_{t}\right)^{-1} \mathbf{E}\left[\zeta_{T} \cdot\left(g^{1}\left(X_{T}^{0, x_{0}}\right)+\int_{0}^{T} h_{1}\left(s, X_{s}^{0, x_{0}}, u_{s}, v_{s}\right) d s\right) \mid \mathcal{F}_{t}\right] \\
& -\int_{0}^{t} h_{1}\left(s, X_{s}^{0, x_{0}}, u_{s}, v_{s}\right) d s .
\end{aligned}
$$

By Corollary 3.1, there exists some $1<p_{0}<2$, such that $\zeta_{T} \in L^{p_{0}}(\mathbf{R})$. Therefore, from Young's inequality, we obtain, for any $1<\bar{q}<p_{0}$,

$$
\begin{aligned}
& \mathbf{E}\left[\left|\zeta_{T} \cdot\left(g^{1}\left(X_{T}^{0, x_{0}}\right)+\int_{0}^{T} h_{1}\left(s, X_{s}^{0, x_{0}}, u_{s}, v_{s}\right) d s\right)\right|^{\bar{q}}\right] \\
\leq & \frac{\bar{q}}{p_{0}} \mathbf{E}\left[\left|\zeta_{T}\right|^{p_{0}}\right]+\frac{p_{0}-\bar{q}}{p_{0}} \mathbf{E}\left[\left|g^{1}\left(X_{T}^{0, x_{0}}\right)+\int_{0}^{T} h_{1}\left(s, X_{s}^{0, x_{0}}, u_{s}, v_{s}\right) d s\right|^{\left.\bar{q} \cdot \frac{p_{0}}{p_{0}-\bar{q}}\right] .}\right.
\end{aligned}
$$

Since $\frac{p_{0}}{\bar{q}}<2$, its conjugate $\frac{p_{0}}{p_{0}-\bar{q}}>2$. Therefore, by the polynomial growth assumptions of $g^{1}$ and $h_{1}$ w.r.t $x$ (Assumption (A2)-(ii),(iii)) and the estimate (3.3), we have

$$
\mathbf{E}\left[\left|g^{1}\left(X_{T}^{0, x_{0}}\right)+\int_{0}^{T} h_{1}\left(s, X_{s}^{0, x_{0}}, u_{s}, v_{s}\right) d s\right|^{\bar{q} \cdot \frac{p_{0}}{p_{0}-\bar{q}}}\right]<\infty .
$$

Then,

$$
\zeta_{T} \cdot\left(g^{1}\left(X_{T}^{0, x_{0}}\right)+\int_{0}^{T} h_{1}\left(s, X_{s}^{0, x_{0}}, u_{s}, v_{s}\right) d s\right) \in L^{\bar{q}}
$$


Stochastics: An International Journal of Probability and Stochastic Processes

Therefore thanks to the representation theorem, there exists a $\mathcal{P}$-measurable and $\mathbf{R}^{m}$-valued process, $\left(\bar{\theta}_{s}\right)_{s \leq T}$ which satisfies,

$$
\mathbf{E}\left[\left(\int_{0}^{T}\left|\bar{\theta}_{s}\right|^{2} d s\right)^{\frac{\bar{q}}{2}}\right]<\infty
$$

such that for any $t \leq T$,

$$
\begin{aligned}
W_{t}^{1,(u, v)}= & \left(\zeta_{t}\right)^{-1} \cdot\left\{\mathbf{E}\left[\zeta_{T} \cdot\left(g^{1}\left(X_{T}^{0, x_{0}}\right)+\int_{0}^{T} h_{1}\left(s, X_{s}^{0, x_{0}}, u_{s}, v_{s}\right) d s\right)\right]+\int_{0}^{t} \bar{\theta}_{s} d B_{s}\right\} \\
& -\int_{0}^{t} h_{1}\left(s, X_{s}^{0, x_{0}}, u_{s}, v_{s}\right) d s \\
\triangleq & \left(\zeta_{t}\right)^{-1} R_{t}-\int_{0}^{t} h_{1}\left(s, X_{s}^{0, x_{0}}, u_{s}, v_{s}\right) d s,
\end{aligned}
$$

where

$$
R_{t} \triangleq \mathbf{E}\left[\zeta_{T} \cdot\left(g^{1}\left(X_{T}^{0, x_{0}}\right)+\int_{0}^{T} h_{1}\left(s, X_{s}^{0, x_{0}}, u_{s}, v_{s}\right)\right)\right]+\int_{0}^{t} \bar{\theta}_{s} d B_{s}, \quad t \leq T .
$$

But for any $s \leq T$,

$$
d \zeta_{s}=\zeta_{s} \sigma^{-1}\left(s, X_{s}^{0, x_{0}}\right) f\left(s, X_{s}^{0, x_{0}}, u_{s}, v_{s}\right) d B_{s} .
$$

Then by Itô's formula we have

$$
\begin{array}{r}
d\left(\zeta_{s}\right)^{-1}=-\left(\zeta_{s}\right)^{-1} \cdot\left\{\sigma^{-1}\left(s, X_{s}^{0, x_{0}}\right) f\left(s, X_{s}^{0, x_{0}}, u_{s}, v_{s}\right) d B_{s}-\right. \\
\left.\left|\sigma^{-1}\left(s, X_{s}^{0, x_{0}}\right) f\left(s, X_{s}^{0, x_{0}}, u_{s}, v_{s}\right)\right|^{2} d s\right\}, \quad s \leq T .
\end{array}
$$

Therefore, $\forall s \leq T$,

$$
\begin{aligned}
d W_{s}^{1,(u, v)}= & -\left(\zeta_{s}\right)^{-1} \cdot\left\{\sigma^{-1}\left(s, X_{s}^{0, x_{0}}\right) f\left(s, X_{s}^{0, x_{0}}, u_{s}, v_{s}\right) d B_{s}\right. \\
& \left.-\left|\sigma^{-1}\left(s, X_{s}^{0, x_{0}}\right) f\left(s, X_{s}^{0, x_{0}}, u_{s}, v_{s}\right)\right|^{2} d s\right\} R_{s} \\
& +\left(\zeta_{s}\right)^{-1} \bar{\theta}_{s} d B_{s}+\left(-\left(\zeta_{s}\right)^{-1} \sigma^{-1}\left(s, X_{s}^{0, x_{0}}\right) f\left(s, X_{s}^{0, x_{0}}, u_{s}, v_{s}\right)\right) \bar{\theta}_{s} d s \\
& -h_{1}\left(s, X_{s}^{0, x_{0}}, u_{s}, v_{s}\right) d s .
\end{aligned}
$$

Next let us define

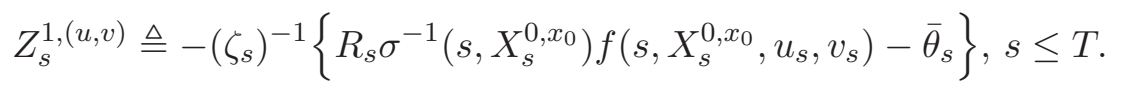

Then it is easy to check that the pair of processes $\left(W_{s}^{1,(u, v)}, Z_{s}^{1,(u, v)}\right)_{s \leq T}$ of (3.8)-(3.11) satisfies the backward equation (3.7).

We now focus on the estimates for the processes $\left(W_{s}^{1,(u, v)}, Z_{s}^{1,(u, v)}\right)_{s \leq T}$. From (3.8), for any $s \leq T$ and $q>1$,

$$
\sup _{0 \leq t \leq T}\left|W_{t}^{1,(u, v)}\right|^{q}=\sup _{0 \leq t \leq T}\left|\mathbf{E}^{(u, v)}\left[g^{1}\left(X_{T}^{0, x_{0}}\right)+\int_{t}^{T} h_{1}\left(s, X_{s}^{0, x_{0}}, u_{s}, v_{s}\right) d s \mid \mathcal{F}_{t}\right]\right|^{q} .
$$

Then by conditional Jensen's inequality we have,

$$
\sup _{0 \leq t \leq T}\left|W_{t}^{1,(u, v)}\right|^{q} \leq \mathbf{E}^{(u, v)}\left[\sup _{0 \leq t \leq T}\left|g^{1}\left(X_{T}^{0, x_{0}}\right)+\int_{t}^{T} h_{1}\left(s, X_{s}^{0, x_{0}}, u_{s}, v_{s}\right) d s\right|^{q} \mid \mathcal{F}_{t}\right] .
$$


Therefore, since $g^{1}$ and $h_{1}$ are of polynomial growth, we have

$$
\begin{aligned}
\mathbf{E}^{(u, v)}\left[\sup _{0 \leq t \leq T}\left|W_{t}^{1,(u, v)}\right|^{q}\right] & \leq \mathbf{E}^{(u, v)}\left[\sup _{0 \leq t \leq T}\left|g^{1}\left(X_{T}^{0, x_{0}}\right)+\int_{t}^{T} h_{1}\left(s, X_{s}^{0, x_{0}}, u_{s}, v_{s}\right) d s\right|^{q}\right] \\
& \leq C \mathbf{E}^{(u, v)}\left[\sup _{0 \leq t \leq T}\left(1+\left|X_{t}^{0, x_{0}}\right|^{\gamma q}\right)\right] \leq C\left(1+\left|x_{0}\right|^{\gamma q}\right)<\infty .
\end{aligned}
$$

Next for each integer $k$, let us define:

$$
\tau_{k}=\inf \left\{s \geq 0, \int_{0}^{s}\left|Z_{s}^{1,(u, v)}\right|^{2} d s \geq k\right\} \wedge T .
$$

The sequence $\left(\tau_{k}\right)_{k} \geq 0$ is of stationary type and converges to $T$. By using Itô's formula with $\left(W_{t \wedge \tau_{k}}^{1,(u, v)}\right)^{2}$ we obtain: $\forall t \leq T$,

$$
\begin{aligned}
& \left|W_{t \wedge \tau_{k}}^{1,(u, v)}\right|^{2}+\int_{t \wedge \tau_{k}}^{\tau_{k}}\left|Z_{s}^{1,(u, v)}\right|^{2} d s \\
& =\left|W_{\tau_{k}}^{1,(u, v)}\right|^{2}+2 \int_{t \wedge \tau_{k}}^{\tau_{k}} W_{s}^{1,(u, v)} h_{1}\left(s, X_{s}^{0, x_{0}}, u_{s}, v_{s}\right) d s-2 \int_{t \wedge \tau_{k}}^{\tau_{k}} W_{s}^{1,(u, v)} Z_{s}^{1,(u, v)} d B_{s}^{(u, v)} .
\end{aligned}
$$

Thus, for $q>1$, taking the expectation of the power $\frac{q}{2}$ of the above equation on both sides and applying Young's inequality, we see that there exists a constant $\underline{\mathrm{C}}$ such that,

$$
\begin{aligned}
\mathbf{E}^{(u, v)}\left[\left(\int_{0}^{\tau_{k}}\left|Z_{s}^{1,(u, v)}\right|^{2} d s\right)^{\frac{q}{2}}\right] \leq & \underline{\mathbf{C}}\left\{\mathbf{E}^{(u, v)}\left[\left|W_{\tau_{k}}^{1,(u, v)}\right|^{q}\right]+\mathbf{E}^{(u, v)}\left[\left(\int_{0}^{\tau_{k}}\left|W_{s}^{1,(u, v)}\right|^{2} d s\right)^{\frac{q}{2}}\right]\right. \\
& +\mathbf{E}^{(u, v)}\left[\left(\int_{0}^{\tau_{k}}\left|h_{1}\left(s, X_{s}^{0, x_{0}}, u_{s}, v_{s}\right)\right|^{2} d s\right)^{\frac{q}{2}}\right] \\
& \left.+\mathbf{E}^{(u, v)}\left[\left|\int_{0}^{\tau_{k}} W_{s}^{1,(u, v)} Z_{s}^{1,(u, v)} d B_{s}^{(u, v)}\right|^{\frac{q}{2}}\right]\right\}
\end{aligned}
$$

Next taking into account the Assumptions (A2)-(ii) and estimate (3.12), one can show that,

$$
\begin{aligned}
& \mathbf{E}^{(u, v)}\left[\left|W_{\tau_{k}}^{1,(u, v)}\right|^{q}\right]+\mathbf{E}^{(u, v)}\left[\left(\int_{0}^{\tau_{k}}\left|W_{s}^{1,(u, v)}\right|^{2} d s\right)^{\frac{q}{2}}\right] \\
& +\mathbf{E}^{(u, v)}\left[\left(\int_{0}^{\tau_{k}}\left|h_{1}\left(s, X_{s}^{0, x_{0}}, u_{s}, v_{s}\right)\right|^{2} d s\right)^{\frac{q}{2}}\right] \\
& \leq \bar{C}\left\{\mathbf{E}^{(u, v)}\left[\sup _{0 \leq s \leq \tau_{k}}\left|W_{s}^{1,(u, v)}\right|^{q}\right]+\mathbf{E}^{(u, v)}\left[\sup _{0 \leq s \leq \tau_{k}}\left(1+\left|X_{s}^{0, x_{0}}\right|^{2 \gamma}\right)^{\frac{q}{2}}\right]\right\} \\
& \leq \bar{C}\left\{\mathbf{E}^{(u, v)}\left[\sup _{0 \leq s \leq T}\left|W_{s}^{1,(u, v)}\right|^{q}\right]+\left\{\mathbf{E}^{(u, v)}\left[\sup _{0 \leq s \leq T}\left(1+\left|X_{s}^{0, x_{0}}\right|^{2 \gamma q}\right)\right]\right\}^{\frac{1}{2}}\right\}<\infty .
\end{aligned}
$$

Meanwhile, it follows from the Burkholder-Davis-Gundy (BDG for short) that there exists a constant $C_{q}$, depending on $q$, such that

$$
\begin{aligned}
\mathbf{E}^{(u, v)} & {\left[\left|\int_{0}^{\tau_{k}} W_{s}^{1,(u, v)} Z_{s}^{1,(u, v)} d B_{s}^{(u, v)}\right|^{\frac{q}{2}}\right] } \\
& \leq C_{q} \mathbf{E}^{(u, v)}\left[\left(\int_{0}^{\tau_{k}}\left|W_{s}^{1,(u, v)}\right|^{2}\left|Z_{s}^{1,(u, v)}\right|^{2} d s\right)^{\frac{q}{4}}\right] \\
& \leq C_{q} \mathbf{E}^{(u, v)}\left[\left(\sup _{0 \leq s \leq \tau_{k}}\left|W_{s}^{1,(u, v)}\right|\right)^{\frac{q}{2}}\left(\int_{0}^{\tau_{k}}\left|Z_{s}^{1,(u, v)}\right|^{2} d s\right)^{\frac{q}{4}}\right]
\end{aligned}
$$


Stochastics: An International Journal of Probability and Stochastic Processes

$$
\leq \frac{C_{q}^{2} \underline{\mathrm{C}}}{2} \mathbf{E}^{(u, v)}\left[\left(\sup _{0 \leq s \leq T}\left|W_{s}^{1,(u, v)}\right|\right)^{q}\right]+\frac{1}{2 \underline{\mathrm{C}}} \mathbf{E}^{(u, v)}\left[\left(\int_{0}^{\tau_{k}}\left|Z_{s}^{1,(u, v)}\right|^{2} d s\right)^{\frac{q}{2}}\right]
$$

where $\underline{\mathrm{C}}$ is the one of (3.13). Going back now to (3.13) and using Fatou's Lemma, we conclude that for any $q>1$,

$$
\mathbf{E}^{(u, v)}\left[\left(\int_{0}^{T}\left|Z_{s}^{1,(u, v)}\right|^{2} d s\right)^{\frac{q}{2}}\right]<\infty .
$$

Estimates (3.12) and (3.14) yield to the conclusion (3.6).

Finally note that, taking $t=0$ in $(3.8)$ we obtain $W_{0}^{1,(u, v)}=J^{1}(u, v)$ since $\mathcal{F}_{0}$ contains only $\mathbf{P}$ and $\mathbf{P}^{(u, v)}$ null sets since those probabilities are equivalent.

Theorem 3.1. Let us assume that:

(i) The Assumptions (A1), (A2) and (A3) are fulfilled;

(ii) There exist two deterministic functions $\varpi^{i}(t, x), i=1,2$, with polynomial growth and two pairs of $\mathcal{P}$-measurable processes $\left(W^{i}, Z^{i}\right), i=1,2$, with values in $\mathbf{R}^{1+m}$ such that: For $i=1,2$,

(a) $\mathbf{P}$-a.s., $\forall s \leq T, W_{s}^{i}=\varpi^{i}\left(s, X_{s}^{0, x}\right)$ and $Z^{i}(\omega):=\left(Z_{t}^{i}(\omega)\right)_{t \leq T}$ is dt-square integrable ;

(b) For any $s \leq T$,

$$
\left\{\begin{aligned}
-d W_{s}^{i} & =H_{i}\left(s, X_{s}^{0, x}, Z_{s}^{i}, u^{*}\left(s, X_{s}^{0, x}, Z_{s}^{1}, Z_{s}^{2}\right), v^{*}\left(s, X_{s}^{0, x}, Z_{s}^{1}, Z_{s}^{2}\right)\right) d s-Z_{s}^{i} d B_{s}, \\
W_{T}^{i} & =g^{i}\left(X_{T}^{0, x}\right) .
\end{aligned}\right.
$$

Then the control $\left(u^{*}\left(s, X_{s}^{0, x}, Z_{s}^{1}, Z_{s}^{2}\right), v^{*}\left(s, X_{s}^{0, x}, Z_{s}^{1}, Z_{s}^{2}\right)\right)_{s \leq T}$ is admissible and a Nash equilibrium point for the NZSDG.

Proof. For $s \leq T$, let us set $u_{s}^{*}=u^{*}\left(s, X_{s}^{0, x}, Z_{s}^{1}, Z_{s}^{2}\right)$ and $v_{s}^{*}=v^{*}\left(s, X_{s}^{0, x}, Z_{s}^{1}, Z_{s}^{2}\right)$, then $\left(u^{*}, v^{*}\right) \in$ $\mathcal{M}$. On the other hand we obviously have, thanks to Proposition $3.1, W_{0}^{1}=J^{1}\left(u^{*}, v^{*}\right)$.

Next let $u$ be an arbitrary element of $\mathcal{M}_{1}$ and let us show that $W^{1} \leq W^{1,\left(u, v^{*}\right)}$, which yields $W_{0}^{1}=J^{1}\left(u^{*}, v^{*}\right) \leq W_{0}^{1,\left(u, v^{*}\right)}=J^{1}\left(u, v^{*}\right)$.

The control $\left(u, v^{*}\right)$ is admissible and thanks to Proposition 3.1, there exists a pair of $\mathcal{P}$ measurable processes $\left(W^{1,\left(u, v^{*}\right)}, Z^{1,\left(u, v^{*}\right)}\right)$ such that for any $q>1$,

$$
\left\{\begin{array}{l}
\mathbf{E}^{\left(u, v^{*}\right)}\left[\sup _{0 \leq t \leq T}\left|W_{t}^{1,\left(u, v^{*}\right)}\right|^{q}+\left(\int_{0}^{T}\left|Z_{s}^{1,\left(u, v^{*}\right)}\right|^{2} d s\right)^{\frac{q}{2}}\right]<\infty \\
W_{t}^{1,\left(u, v^{*}\right)}=g^{1}\left(X_{T}^{0, x}\right)+\int_{t}^{T} H_{1}\left(s, X_{s}^{0, x}, Z_{s}^{1,\left(u, v^{*}\right)}, u_{s}, v_{s}^{*}\right) d s-\int_{t}^{T} Z_{s}^{1,\left(u, v^{*}\right)} d B_{s}, \forall t \leq T .
\end{array}\right.
$$

Afterwards, we aim to compare $W^{1}$ and $W^{1,\left(u, v^{*}\right)}$. So let us denote by

$$
\triangle W=W^{1}-W^{1,\left(u, v^{*}\right)} \text { and } \triangle Z=Z^{1}-Z^{1,\left(u, v^{*}\right)} .
$$

For $k \geq 0$, we define the stopping time $\tau_{k}$ as follows:

$$
\tau_{k}:=\inf \left\{s \geq 0,\left|\triangle W_{s}\right|+\int_{0}^{s}\left|\triangle Z_{r}\right|^{2} d r \geq k\right\} \wedge T .
$$

The sequence of stopping times $\left(\tau_{k}\right)_{k \geq 0}$ is of stationary type and converges to $T$. Next applying Itô-Meyer formula to $\left|(\triangle W)^{+}\right|^{q}(q>1)$ (see Theorem 71, P.Protter, [27], pp.221), between $t \wedge \tau_{k}$ 
Stochastics: An International Journal of Probability and Stochastic Processes

and $\tau_{k}$, we obtain: $\forall t \leq T$,

$$
\begin{aligned}
\left|\left(\triangle W_{t \wedge \tau_{k}}\right)^{+}\right|^{q}+c(q) \int_{t \wedge \tau_{k}}^{\tau_{k}}\left|\left(\triangle W_{s}\right)^{+}\right|^{q-2} 1_{\triangle W_{s}>0}\left|\triangle Z_{s}\right|^{2} d s & \left|\left(\triangle W_{\tau_{k}}\right)^{+}\right|^{q}+q \int_{t \wedge \tau_{k}}^{\tau_{k}}\left|\left(\triangle W_{s}\right)^{+}\right|^{q-1} 1_{\triangle W_{s}>0}\left(H_{1}\left(s, X_{s}^{0, x}, Z_{s}^{1}, u_{s}^{*}, v_{s}^{*}\right)-\right. \\
& \left.H_{1}\left(s, X_{s}^{0, x}, Z_{s}^{\left(u, v^{*}\right)}, u_{s}, v_{s}^{*}\right)\right) d s-q \int_{t \wedge \tau_{k}}^{\tau_{k}}\left|\left(\triangle W_{s}\right)^{+}\right|^{q-1} 1_{\triangle W_{s}>0} \triangle Z_{s} d B_{s} \\
= & \left|\left(\triangle W_{\tau_{k}}\right)^{+}\right|^{q}+q \int_{t \wedge \tau_{k}}^{\tau_{k}}\left|\left(\triangle W_{s}\right)^{+}\right|^{q-1} 1_{\triangle W_{s}>0}\left(H_{1}\left(s, X_{s}^{0, x}, Z_{s}^{1}, u_{s}^{*}, v_{s}^{*}\right)-\right. \\
& \left.H_{1}\left(s, X_{s}^{0, x}, Z_{s}^{1}, u_{s}, v_{s}^{*}\right)+H_{1}\left(s, X_{s}^{0, x}, Z_{s}^{1}, u_{s}, v_{s}^{*}\right)-H_{1}\left(s, X_{s}^{0, x}, Z_{s}^{\left(u, v^{*}\right)}, u_{s}, v_{s}^{*}\right)\right) d s \\
& -q \int_{t \wedge \tau_{k}}^{\tau_{k}}\left|\left(\triangle W_{s}\right)^{+}\right|^{q-1} 1_{\triangle W_{s}>0} \triangle Z_{s} d B_{s},
\end{aligned}
$$

where $c(q)=\frac{q(q-1)}{2}$. Considering now the generalized Isaacs' Assumption (A3), we have that,

$$
H_{1}\left(s, X_{s}^{0, x}, Z_{s}^{1}, u_{s}^{*}, v_{s}^{*}\right)-H_{1}\left(s, X_{s}^{0, x}, Z_{s}^{1}, u_{s}, v_{s}^{*}\right) \leq 0, \forall s \leq T .
$$

Therefore,

$$
\begin{aligned}
\left|\left(\Delta W_{t \wedge \tau_{k}}\right)^{+}\right|^{q}+c(q) \int_{t \wedge \tau_{k}}^{\tau_{k}}\left|\left(\triangle W_{s}\right)^{+}\right|^{q-2} 1_{\triangle W_{s}>0}\left|\triangle Z_{s}\right|^{2} d s \\
\leq\left|\left(\triangle W_{\tau_{k}}\right)^{+}\right|^{q}+q \int_{t \wedge \tau_{k}}^{\tau_{k}}\left|\left(\triangle W_{s}\right)^{+}\right|^{q-1} 1_{\triangle W_{s}>0} \triangle Z_{s} \sigma^{-1}\left(s, X_{s}^{0, x}\right) f\left(s, X_{s}^{0, x}, u_{s}, v_{s}^{*}\right) d s \\
\quad-q \int_{t \wedge \tau_{k}}^{\tau_{k}}\left|\left(\triangle W_{s}\right)^{+}\right|^{q-1} 1_{\triangle W_{s}>0} \triangle Z_{s} d B_{s} \\
=\left|\left(\triangle W_{\tau_{k}}\right)^{+}\right|^{q}-q \int_{t \wedge \tau_{k}}^{\tau_{k}}\left|\left(\triangle W_{s}\right)^{+}\right|^{q-1} 1_{\triangle W_{s}>0} \triangle Z_{s} d B_{s}^{\left(u, v^{*}\right)},
\end{aligned}
$$

where $B^{\left(u, v^{*}\right)}=\left(B_{t}-\int_{0}^{t} \sigma^{-1}\left(s, X_{s}^{0, x}\right) f\left(s, X_{s}^{0, x}, u_{s}, v_{s}^{*}\right) d s\right)_{t \leq T}$ is an $\left(\mathcal{F}_{t}^{0}, \mathbf{P}^{\left(u, v^{*}\right)}\right)$-Brownian motion. Then for any $t \leq T$,

$$
\left|\left(\triangle W_{t \wedge \tau_{k}}\right)^{+}\right|^{q} \leq\left|\left(\triangle W_{\tau_{k}}\right)^{+}\right|^{q}-q \int_{t \wedge \tau_{k}}^{\tau_{k}}\left|\left(\triangle W_{s}\right)^{+}\right|^{q-1} 1_{\triangle W_{s}>0} \triangle Z_{s} d B_{s}^{\left(u, v^{*}\right)} .
$$

By definition of the stopping time $\tau_{k}$, we have

$$
\mathbf{E}^{\left(u, v^{*}\right)}\left[\int_{t \wedge \tau_{k}}^{\tau_{k}}\left|\left(\triangle W_{s}\right)^{+}\right|^{q-1} 1_{\triangle W_{s}>0} \triangle Z_{s} d B_{s}^{\left(u, v^{*}\right)}\right]=0 .
$$

Then taking expectation on both sides of (3.17) we obtain:

$$
\mathbf{E}^{\left(u, v^{*}\right)}\left[\left|\left(\triangle W_{t \wedge \tau_{k}}\right)^{+}\right|^{q}\right] \leq \mathbf{E}^{\left(u, v^{*}\right)}\left[\left|\left(W_{\tau_{k}}^{1}-W_{\tau_{k}}^{1,\left(u, v^{*}\right)}\right)^{+}\right|^{q}\right] .
$$

Next taking into account (3.16) and the fact that $W^{1}$ has a representation through $\varpi^{1}$ which is deterministic and of polynomial growth and finally (3.4), we deduce that

$$
\mathbf{E}^{\left(u, v^{*}\right)}\left[\sup _{s \leq T}\left(\left|W_{s}^{1,\left(u, v^{*}\right)}\right|+\left|W_{s}^{1}\right|\right)^{q}\right]<\infty .
$$


As the sequence $\left(\left(W_{\tau_{k}}^{1}-W_{\tau_{k}}^{1,\left(u, v^{*}\right)}\right)^{+}\right)_{k}$ converges to 0 as $k \rightarrow \infty, \mathbf{P}^{\left(u, v^{*}\right)}$-a.s., then it converges also to 0 in $L^{1}\left(d \mathbf{P}^{\left(u, v^{*}\right)}\right)$ since it is uniformly integrable thanks to (3.19). Taking now the limit w.r.t. $k$ on both sides of (3.18) and finally by Fatou's Lemma we deduce that:

$$
\mathbf{E}^{\left(u, v^{*}\right)}\left[\triangle W_{t}^{+}\right]=0, \forall t \leq T,
$$

which implies that $W^{1} \leq W^{1,\left(u, v^{*}\right)}$, $\mathbf{P}$-a.s., since the probabilities $\mathbf{P}^{\left(u, v^{*}\right)}$ and $\mathbf{P}$ are equivalent. Thus $W_{0}^{1}=J^{1}\left(u^{*}, v^{*}\right) \leq W_{0}^{1,\left(u, v^{*}\right)}=J^{1}\left(u, v^{*}\right)$.

In the same way one can show that if $v$ is an arbitrary element of $\mathcal{M}_{2}$ then $W_{0}^{2}=J^{2}\left(u^{*}, v^{*}\right) \leq$ $W_{0}^{2,\left(u^{*}, v\right)}=J^{2}\left(u^{*}, v\right)$. Henceforth $\left(u^{*}, v^{*}\right)$ is a Nash equilibrium point for the NZSDG.

Now, the main emphasis is placed on the existence of a solution for the BSDE (3.15) with its properties.

\section{Existence of solutions for markovian BSDEs related to differ- ential games}

\subsection{Deterministic representation}

Let $\ell$ be an integer and let us consider $\bar{f}$ (resp. $\bar{g}$ ) a Borel measurable function from $[0, T] \times$ $\mathbf{R}^{m+\ell+\ell \times m}$ (resp. $\mathbf{R}^{m}$ ) into $\mathbf{R}^{\ell}$ (resp. $\mathbf{R}^{\ell}$ ) such that:

(a) For any fixed $(t, x) \in[0, T] \times \mathbf{R}^{m}$, the mapping $(y, z) \in \mathbf{R}^{\ell+\ell \times m} \longmapsto \bar{f}(t, x, y, z)$ is uniformly Lipschitz ;

(b) There exist real constants $C$ and $p>0$ such that

$$
|\bar{f}(t, x, y, z)|+|\bar{g}(x)| \leq C\left(1+|x|^{p}\right), \forall(t, x, y, z) \in[0, T] \times \mathbf{R}^{m+\ell+\ell \times m} .
$$

Then we have the following result by El Karoui et al. [8] related to representation of solutions of BSDEs through deterministic functions in the Markovian framework of randomness.

Proposition 4.1. Assume that (A1), (i)-(ii) and (A4) are fulfilled. Let $(t, x) \in[0, T] \times \mathbf{R}^{m}$ be fixed and $\left(\theta_{s}^{t, x}\right)_{t \leq s \leq T}$ be the solution of $S D E$ (3.1). Let $\left(y_{s}^{t, x}, z_{s}^{t, x}\right)_{t \leq s \leq T}$ be the solution of the following BSDE:

$$
\left\{\begin{array}{l}
y^{t, x} \in \mathcal{S}_{t, T}^{2}\left(\mathbf{R}^{\ell}\right), z^{t, x} \in \mathcal{H}_{t, T}^{2}\left(\mathbf{R}^{\ell \times m}\right) \\
-d y_{s}^{t, x}=\bar{f}\left(s, \theta_{s}^{t, x}, y_{s}^{t, x}, z_{s}^{t, x}\right) d s-z_{s}^{t, x} d B_{s}, s \in[t, T] \\
y_{T}=\bar{g}\left(\theta_{T}^{t, x}\right) .
\end{array}\right.
$$

Then there exists a pair of measurable and deterministic applications $\varpi:[0, T] \times \mathbf{R}^{m} \rightarrow \mathbf{R}^{\ell}$ and $v:[0, T] \times \mathbf{R}^{m} \rightarrow \mathbf{R}^{\ell \times d}$ such that,

$$
\mathbf{P}-a . s ., \forall t \leq s \leq T, \quad y_{s}^{t, x}=\varpi\left(s, \theta_{s}^{t, x}\right) \text { and } z_{s}^{t, x}=v\left(s, \theta_{s}^{t, x}\right) .
$$

Moreover,

(i) $\forall(t, x) \in[0, T] \times \mathbf{R}^{m}, \varpi(t, x)=\mathbf{E}\left[\int_{t}^{T} \bar{f}\left(r, \theta_{r}^{t, x}, y_{r}^{t, x}, z_{r}^{t, x}\right) d r+\bar{g}\left(\theta_{T}^{t, x}\right)\right]$;

(ii) For any other $\left(t_{1}, x_{1}\right) \in[0, T] \times \mathbf{R}^{m}$, the process $\left(\varpi\left(s, \theta_{s}^{t_{1}, x_{1}}\right), v\left(s, \theta_{s}^{t_{1}, x_{1}}\right)\right)_{t_{1} \leq s \leq T}$ is the unique solution in $\mathcal{S}_{t_{1}, T}^{2}\left(\mathbf{R}^{\ell}\right) \times \mathcal{H}_{t_{1}, T}^{2}\left(\mathbf{R}^{\ell \times m}\right)$ of the BSDE associated with the coefficients $\left(\bar{f}\left(s, \theta_{s}^{t_{1}, x_{1}}, y, z\right), \bar{g}\left(\theta_{T}^{t_{1}, x_{1}}\right)\right)$ in the time interval $\left[t_{1}, T\right]$. 
Stochastics: An International Journal of Probability and Stochastic Processes

We next recall the notion of domination which is important in order to show that equation (3.15) has a solution.

\section{Definition 4.1. : $L^{q}$-Domination condition}

Let $q \in] 1, \infty\left[\right.$ be fixed. For a given $t_{1} \in[0, T]$, a family of probability measures $\left\{\nu_{1}(s, d x), s \in\right.$ $\left.\left[t_{1}, T\right]\right\}$ defined on $\mathbf{R}^{m}$ is said to be $L^{q}$ - dominated by another family of probability measures $\left\{\nu_{0}(s, d x), s \in\left[t_{1}, T\right]\right\}$, if for any $\delta \in\left(0, T-t_{1}\right]$, there exists an application $\phi_{t_{1}}^{\delta}:\left[t_{1}+\delta, T\right] \times \mathbf{R}^{m} \rightarrow$ $\mathbf{R}^{+}$such that:

(i) $\nu_{1}(s, d x) d s=\phi_{t_{1}}^{\delta}(s, x) \nu_{0}(s, d x) d s$ on $\left[t_{1}+\delta, T\right] \times \mathbf{R}^{m}$.

(ii) $\forall k \geq 1, \phi_{t_{1}}^{\delta}(s, x) \in L^{q}\left(\left[t_{1}+\delta, T\right] \times[-k, k]^{m} ; \nu_{0}(s, d x) d s\right)$.

We then have:

Lemma 4.1. Assume (A1) and (A4) fulfilled and the drift term $b(t, x)$ of $S D E$ (3.1) is bounded. Let $q \in] 1, \infty$ [ be fixed, $\left(t_{0}, x_{0}\right) \in[0, T] \times \mathbf{R}^{m}$ and let $\left(\theta_{s}^{t_{0}, x_{0}}\right)_{t_{0} \leq s \leq T}$ be the solution of SDE (3.1). Then for any $s \in\left(t_{0}, T\right]$, the law $\bar{\mu}\left(t_{0}, x_{0} ; s, d x\right)$ of $\theta_{s}^{t_{0}, x_{0}}$ has a density function $\rho_{t_{0}, x_{0}}(s, x)$, w.r.t. Lebesgue measure $d x$, which satisfies the following estimate: $\forall(s, x) \in\left(t_{0}, T\right] \times \mathbf{R}^{m}$,

$$
\varrho_{1}\left(s-t_{0}\right)^{-\frac{m}{2}} \exp \left[-\frac{\Lambda\left|x-x_{0}\right|^{2}}{s-t_{0}}\right] \leq \rho_{t_{0}, x_{0}}(s, x) \leq \varrho_{2}\left(s-t_{0}\right)^{-\frac{m}{2}} \exp \left[-\frac{\lambda\left|x-x_{0}\right|^{2}}{s-t_{0}}\right]
$$

where $\varrho_{1}, \varrho_{2}, \Lambda, \lambda$ are real constants such that $0 \leq \varrho_{1} \leq \varrho_{2}$ and $0 \leq \lambda \leq \Lambda$. Additionally for any $\left(t_{1}, x_{1}\right) \in\left[t_{0}, T\right] \times \mathbf{R}^{m}$, the family of laws $\left\{\bar{\mu}\left(t_{1}, x_{1} ; s, d x\right), s \in\left[t_{1}, T\right]\right\}$ is $L^{q}$-dominated by $\bar{\mu}\left(t_{0}, x_{0} ; s, d x\right)$.

Proof: Since $\sigma$ satisfies (2.1) and $b$ is bounded, then by Aronson's result (see [1]), the law $\bar{\mu}\left(t_{0}, x_{0} ; s, d x\right)$ of $\left.\left.\theta_{s}^{t_{0}, x_{0}}, s \in\right] t_{0}, T\right]$, has a density function $\rho_{t_{0}, x_{0}}(s, x)$ which satisfies estimate (4.1).

Let us focus on the second claim of the lemma. Let $\left(t_{1}, x_{1}\right) \in\left[t_{0}, T\right] \times \mathbf{R}^{m}$ and $s \in\left(t_{1}, T\right]$. Then,

$$
\rho_{t_{1}, x_{1}}(s, x)=\left[\rho_{t_{1}, x_{1}}(s, x) \rho_{t_{0}, x_{0}}^{-1}(s, x)\right] \rho_{t_{0}, x_{0}}(s, x)=\phi_{t_{1}, x_{1}}(s, x) \rho_{t_{0}, x_{0}}(s, x)
$$

with

$$
\phi_{t_{1}, x_{1}}(s, x)=\left[\rho_{t_{1}, x_{1}}(s, x) \rho_{t_{0}, x_{0}}^{-1}(s, x)\right],(s, x) \in\left(t_{1}, T\right] \times \mathbf{R}^{m} .
$$

For any $\delta \in\left(0, T-t_{1}\right], \phi_{t_{1}, x_{1}}$ is defined on $\left[t_{1}+\delta, T\right]$. Moreover for any $(s, x) \in\left[t_{1}+\delta, T\right] \times \mathbf{R}^{m}$ it holds,

$$
\begin{aligned}
\bar{\mu}\left(t_{1}, x_{1} ; s, d x\right) d s & =\rho_{t_{1}, x_{1}}(s, x) d x d s \\
& =\phi_{t_{1}, x_{1}}(s, x) \rho_{t_{0}, x_{0}}(s, x) d x d s \\
& =\phi_{t_{1}, x_{1}}(s, x) \bar{\mu}\left(t_{0}, x_{0} ; s, d x\right) d s .
\end{aligned}
$$

Next by (4.1), for any $(s, x) \in\left[t_{1}+\delta, T\right] \times \mathbf{R}^{m}$,

$$
0 \leq \phi_{t_{1}, x_{1}}(s, x) \leq \frac{\varrho_{2}\left(s-t_{1}\right)^{-\frac{m}{2}}}{\varrho_{1}\left(s-t_{0}\right)^{-\frac{m}{2}}} \exp \left[\frac{\Lambda\left|x-x_{0}\right|^{2}}{s-t_{0}}-\frac{\lambda\left|x-x_{1}\right|^{2}}{s-t_{1}}\right]:=\Phi_{t_{1}, x_{1}}(s, x) .
$$

It follows that for any $k \geq 0$, the function $\Phi_{t_{1}, x_{1}}(s, x)$ is bounded on $\left[t_{1}+\delta, T\right] \times[-k, k]^{m}$ by a constant $\kappa$ which depends on $t_{0}, t_{1}, \delta, \Lambda, \lambda, k$ and $x_{0}$. Next let $q \in(1, \infty)$, then,

$$
\begin{aligned}
\int_{t_{1}+\delta}^{T} \int_{[-k, k]^{m}} \Phi_{t_{1}, x_{1}}(s, x)^{q} \bar{\mu}\left(t_{0}, x_{0} ; s, d x\right) d s & \leq \kappa^{q} \int_{t_{1}+\delta}^{T} \int_{[-k, k]^{m}} \bar{\mu}\left(t_{0}, x_{0} ; s, d x\right) d s \\
& =\kappa^{q} \int_{t_{1}+\delta}^{T} d s \mathbf{E}\left[1_{[-k, k]^{m}}\left(\theta_{s}^{t_{0}, x_{0}}\right)\right] \leq \kappa^{q} T .
\end{aligned}
$$


Stochastics: An International Journal of Probability and Stochastic Processes

Thus $\Phi$ and then $\phi$ belong to $L^{q}\left(\left[t_{1}+\delta, T\right] \times[-k, k]^{m} ; \nu_{0}(s, d x) d s\right)$. It follows that the family of measures $\left\{\bar{\mu}\left(t_{1}, x_{1} ; s, d x\right), s \in\left[t_{1}, T\right]\right\}$ is $L^{q}$-dominated by $\bar{\mu}\left(t_{0}, x_{0} ; s, d x\right)$.

As a by-product we have:

Corollary 4.1. Let $x_{0} \in \mathbf{R}^{m},(t, x) \in[0, T] \times \mathbf{R}^{m}, s \in(t, T]$ and $\mu(t, x ; s, d y)$ the law of $X_{s}^{t, x}$, i.e.,

$$
\forall A \in \mathcal{B}\left(\mathbf{R}^{m}\right), \mu(t, x ; s, A)=\mathbf{P}\left(X_{s}^{t, x} \in A\right) .
$$

Under (A1) on $\sigma$, for any $q \in(1, \infty)$, the family of laws $\{\mu(t, x ; s, d y), s \in[t, T]\}$ is $L^{q}$-dominated by $\left\{\mu\left(0, x_{0} ; s, d y\right), s \in[t, T]\right\}$.

\subsection{The main result}

We are now ready to provide a solution for BSDE (3.15) which satisfies the representation property via deterministic functions with polynomial growth.

Theorem 4.1. Let $x_{0} \in \mathbf{R}^{m}$ be fixed. Then under the Assumptions (A1), (A2) and (A3), there exist:

(i) Two pairs of $\mathcal{P}$-measurable processes $\left(W_{s}^{i}, Z_{s}^{i}\right)_{s \leq T}, i=1,2$, such that: $\forall i \in\{1,2\}$,

$$
\left\{\begin{array}{l}
\mathbf{P}-\text { a.s., } Z^{i}(\omega)=\left(Z_{s}^{i}(\omega)\right)_{s \leq T} \text { is dt-square integrable; } \\
-d W_{s}^{i}=H_{i}\left(s, X_{s}^{0, x_{0}}, Z_{s}^{i}, u_{1}^{*}\left(s, X_{s}^{0, x_{0}}, Z_{s}^{1}, Z_{s}^{2}\right), u_{2}^{*}\left(s, X_{s}^{0, x_{0}}, Z_{s}^{1}, Z_{s}^{2}\right)\right) d s-Z_{s}^{i} d B_{s}, \\
\quad \forall s \leq T ; \\
W_{T}^{i}=g^{i}\left(X_{T}^{0, x_{0}}\right) .
\end{array}\right.
$$

(ii) Two measurable deterministic functions $\varpi^{i}, i=1,2$ with polynomial growth defined from $[0, T] \times \mathbf{R}^{m}$ into $\mathbf{R}$ such that:

$$
\forall i=1,2, W_{s}^{i}=\varpi^{i}\left(s, X_{s}^{0, x_{0}}\right), \forall s \in[0, T] .
$$

Proof. It will be divided into five steps. We first construct an approximating sequence of BSDEs which have solutions according to Proposition 4.1, we then provide a priori estimates of the solutions of those BSDEs. Finally we prove that those solutions are convergent (at least for a subsequence) and the limit is a solution for the BSDE (4.2).

Step 1: Construction of the approximating sequence $\left(W_{s}^{i n ;(t, x)}, Z_{s}^{i n ;(t, x)}\right)_{s \leq T}, n \geq 1, i=1,2$.

Let $\xi$ be an element of $C^{\infty}\left(\mathbf{R}^{2 m}, \mathbf{R}\right)$ with compact support and satisfying

$$
\int_{\mathbf{R}^{2 m}} \xi(y, z) d y d z=1 .
$$

For $n \geq 1, i=1,2$ and $\left(t, x, z^{1}, z^{2}\right) \in[0, T] \times \mathbf{R}^{3 m}$, we set

$$
\begin{aligned}
& \underline{\mathrm{H}}_{i}^{n}\left(t, x, z^{1},\left(u_{1}^{*}, u_{2}^{*}\right)\left(t, x, z^{1}, z^{2}\right)\right) \\
& \quad=\int_{\mathbf{R}^{2 d}} n^{2} H_{i}\left(t, \varphi_{n}(x), y,\left(u_{1}^{*}, u_{2}^{*}\right)\left(t, \varphi_{n}(x), y, z\right)\right) \xi\left(n\left(z^{1}-y\right), n\left(z^{2}-z\right)\right) d y d z,
\end{aligned}
$$

where for any $x=\left(x_{j}\right)_{1 \leq j \leq m} \in \mathbf{R}^{m}, \varphi_{n}(x):=\left(\left(x_{j} \vee(-n)\right) \wedge n\right)_{1 \leq j \leq m}$.

We next define $\psi \in C^{\infty}\left(\mathbf{R}^{2 m}, \mathbf{R}\right)$ satisfying

$$
\psi(y, z)= \begin{cases}1 \text { if } & |y|^{2}+|z|^{2} \leq 1 \\ 0 \text { if } & |y|^{2}+|z|^{2} \geq 4\end{cases}
$$


Then, for $i=1,2$, the measurable functions $H_{i}^{n}, n \geq 1$, defined as follows:

$$
\begin{gathered}
H_{i}^{n}\left(t, x, z^{1}, z^{2}\right):=\psi\left(\frac{z^{1}}{n}, \frac{z^{2}}{n}\right) \underline{H}_{i}^{n}\left(t, x, z^{1},\left(u_{1}^{*}, u_{2}^{*}\right)\left(t, x, z^{1}, z^{2}\right)\right), \\
\left(t, x, z^{1}, z^{2}\right) \in[0, T] \times \mathbf{R}^{3 m}
\end{gathered}
$$

satisfy the following properties:

(a) $H_{i}^{n}$ is uniformly Lipschitz w.r.t $\left(z^{1}, z^{2}\right)$;

(b) $\left|H_{i}^{n}\left(t, x, z^{1}, z^{2}\right)\right| \leq C\left(1+\left|\varphi_{n}(x)\right|\right)\left|z^{i}\right|+C\left(1+\left|\varphi_{n}(x)\right|^{\gamma}\right)$.

(c) $\left|H_{i}^{n}\left(t, x, z^{1}, z^{2}\right)\right| \leq c_{n}$, for any $\left(t, x, z^{1}, z^{2}\right)$.

(d) For any $(t, x) \in[0, T] \times \mathbf{R}^{m}$ and $K$ a compact subset of $\mathbf{R}^{2 m}$,

$$
\sup _{\left(z^{1}, z^{2}\right) \in K}\left|H_{i}^{n}\left(t, x, z^{1}, z^{2}\right)-H_{i}\left(t, x, z^{1},\left(u_{1}^{*}, u_{2}^{*}\right)\left(t, x, z^{1}, z^{2}\right)\right)\right| \rightarrow 0 \text {, as } n \rightarrow \infty .
$$

Let us notice that (b) is valid since $u_{1}^{*}$ and $u_{2}^{*}$ take their values in compact sets.

The constant $\gamma$, which we can choose greater than 1 , is the one of polynomial growth of $h_{i}$, $i=1,2$. Therefore, from points (a) and (c) and Proposition 4.1, for each $n \geq 1, i=1,2$ and $(t, x) \in[0, T] \times \mathbf{R}^{m}$, there exist solutions $\left(W_{s}^{i n ;(t, x)}, Z_{s}^{i n ;(t, x)}\right)_{s \leq T}$ in $\mathcal{S}_{t, T}^{2}(\mathbf{R}) \times \mathcal{H}_{t, T}^{2}\left(\mathbf{R}^{m}\right)$ such that for any $s \in[t, T]$,

$$
W_{s}^{i n ;(t, x)}=g^{i}\left(X_{T}^{t, x}\right)+\int_{s}^{T} H_{i}^{n}\left(r, X_{r}^{t, x}, Z_{r}^{1 n ;(t, x)}, Z_{r}^{2 n ;(t, x)}\right) d r-\int_{s}^{T} Z_{r}^{i n ;(t, x)} d B_{r} .
$$

Then, by Proposition 4.1, for $i=1,2$, there exists a sequence of measurable deterministic functions $\varpi^{i n}:[0, T] \times \mathbf{R}^{m} \rightarrow \mathbf{R}$ and $v^{i n}:[0, T] \times \mathbf{R}^{m} \rightarrow \mathbf{R}^{m}$ such that

$$
\forall s \in[t, T], W_{s}^{i n ;(t, x)}=\varpi^{i n}\left(s, X_{s}^{t, x}\right) \quad \text { and } \quad Z_{s}^{i n ;(t, x)}=v^{i n}\left(s, X_{s}^{t, x}\right) .
$$

Moreover, for $i=1,2$ and $n \geq 1$, $\varpi^{i n}$ satisfies

$$
\varpi^{i n}(t, x)=\mathbf{E}\left[g^{i}\left(X_{T}^{t, x}\right)+\int_{t}^{T} F^{i n}\left(s, X_{s}^{t, x}\right) d s\right], \quad \forall(t, x) \in[0, T] \times \mathbf{R}^{m},
$$

with

$$
F^{i n}(t, x)=H_{i}^{n}\left(t, x, v^{1 n}(t, x), v^{2 n}(t, x)\right),(t, x) \in[0, T] \times \mathbf{R}^{m} .
$$

Step 2: The deterministic functions $\varpi^{i n}$ are of polynomial growth uniformly w.r.t. $n$, i.e., there exist two constants $C$ and $\lambda$ such that for any $n \geq 1, i=1,2$,

$$
\forall(t, x) \in[0, T] \times \mathbf{R}^{m},\left|\varpi^{i n}(t, x)\right| \leq C\left(1+|x|^{\lambda}\right) .
$$

We will deal with the case of index $i=1$, the case of $i=2$ can be treated in the same way. For each $n \geq 1$, let us consider the following BSDE: $\forall s \in[t, T]$,

$$
\left\{\begin{array}{l}
(\bar{W}, \bar{Z}) \in \mathcal{S}_{t, T}^{2}(\mathbf{R}) \times \mathcal{H}_{t, T}^{2}\left(\mathbf{R}^{m}\right) ; \\
\bar{W}_{s}^{1 n}=g^{1}\left(X_{T}^{t, x}\right)+\int_{s}^{T} C\left(1+\left|\varphi_{n}\left(X_{r}^{t, x}\right)\right|\right)\left|\bar{Z}_{r}^{1 n}\right|+C\left(1+\left|\varphi_{n}\left(X_{r}^{t, x}\right)\right|^{\gamma}\right) d r-\int_{s}^{T} \bar{Z}_{r}^{1 n} d B_{r} .
\end{array}\right.
$$

For any $x \in \mathbf{R}^{m}$ and $n \geq 1$, the function $z^{1} \in \mathbf{R}^{m} \longmapsto C\left(1+\left|\varphi_{n}\left(X_{s}^{t, x}\right)\right|\right)\left|z^{1}\right|+C\left(1+\left|\varphi_{n}\left(X_{s}^{t, x}\right)\right|^{\gamma}\right)$ is Lipschitz continuous. Then the 
solution $\left(\bar{W}^{1 n}, \bar{Z}^{1 n}\right)$ exists and is unique. Moreover through an adaptation of the result given in (El Karoui and al.,1997,[8]), we can infer the existence of deterministic measurable function $\bar{\varpi}^{1 n}:[0, T] \times \mathbf{R}^{m} \rightarrow \mathbf{R}$ such that, for any $s \in[t, T]$,

$$
\bar{W}_{s}^{1 n}=\bar{\varpi}^{1 n}\left(s, X_{s}^{t, x}\right) .
$$

Next let us consider the process

$$
B_{s}^{n}=B_{s}-\int_{0}^{s} 1_{[t, T]}(r) C\left(1+\left|\varphi_{n}\left(X_{r}^{t, x}\right)\right|\right) \operatorname{sign}\left(\bar{Z}_{r}^{1 n}\right) d r, 0 \leq s \leq T,
$$

which is, thanks to Girsanov's Theorem, a Brownian motion under the probability $\mathbf{P}^{n}$ on $(\Omega, \mathcal{F})$ whose density with respect to $\mathbf{P}$ is

$$
\zeta_{T}\left\{C\left(1+\left|\varphi_{n}\left(X_{s}^{t, x}\right)\right|\right) \operatorname{sign}\left(\bar{Z}_{s}^{1 n}\right) 1_{[t, T]}(s)\right\},
$$

where for any $z=\left(z^{i}\right)_{i=1, \ldots, d} \in \mathbf{R}^{m}, \operatorname{sign}(z)=\left(1_{\left[\left|z^{i}\right| \neq 0\right]} \frac{z^{i}}{\left|z^{i}\right|}\right)_{i=1, \ldots, d}$ and $\zeta_{T}(\cdot)$ is defined by $(2.3)$. Then (4.6) becomes

$$
\bar{W}_{s}^{1 n}=g^{1}\left(X_{T}^{t, x}\right)+\int_{s}^{T} C\left(1+\left|\varphi_{n}\left(X_{r}^{t, x}\right)\right|^{\gamma}\right) d r-\int_{s}^{T} \bar{Z}_{r}^{1 n} d B_{r}^{n}, \quad 0 \leq s \leq T .
$$

Therefore, taking into account of (4.7), we deduce,

$$
\bar{\varpi}^{1 n}(t, x)=\mathbf{E}^{n}\left[g^{1}\left(X_{T}^{t, x}\right)+\int_{t}^{T} C\left(1+\left|\varphi_{n}\left(X_{s}^{t, x}\right)\right|^{\gamma}\right) d s \mid \mathcal{F}_{t}\right],
$$

where $\mathbf{E}^{n}$ is the expectation under probability $\mathbf{P}^{n}$. Taking the expectation on both sides under the probability $\mathbf{P}^{n}$ and considering $\bar{\varpi}^{1 n}(t, x)$ is deterministic, one obtains,

$$
\bar{\varpi}^{1 n}(t, x)=\mathbf{E}^{n}\left[g^{1}\left(X_{T}^{t, x}\right)+\int_{t}^{T} C\left(1+\left|\varphi_{n}\left(X_{s}^{t, x}\right)\right|^{\gamma}\right) d s\right] .
$$

Then by the Assumption (A2)-(iii) we have: $\forall(t, x) \in[0, T] \times \mathbf{R}^{m}$,

$$
\begin{aligned}
\left|\bar{\varpi}^{1 n}(t, x)\right| & \leq C \mathbf{E}^{n}\left[\sup _{0 \leq s \leq T}\left(1+\left|X_{s}^{t, x}\right|^{\gamma}\right)\right] \\
& =C \mathbf{E}\left[\left(\sup _{0 \leq s \leq T}\left(1+\left|X_{s}^{t, x}\right|^{\gamma}\right)\right)\left(\zeta_{T}\left(C\left(1+\left|\varphi_{n}\left(X_{s}^{t, x}\right)\right|\right) \operatorname{sign}\left(\bar{Z}_{s}^{1 n}\right)\right)\right)\right] .
\end{aligned}
$$

By Lemma 3.1, there exists some $1<p_{0}<2$ (which does not depend on $(t, x)$ ), such that,

$$
\mathbf{E}\left[\left(\zeta_{T}\left(C\left(1+\left|\varphi_{n}\left(X_{s}^{t, x}\right)\right|\right) \operatorname{sign}\left(\tilde{Z}_{s}^{1 n}\right)\right)\right)^{p_{0}}\right]<\infty
$$

Then thanks to Young's inequality, we obtain,

$$
\begin{aligned}
& \left|\bar{\varpi}^{1 n}(t, x)\right| \\
& \leq C\left\{\mathbf{E}\left[\sup _{0 \leq s \leq T}\left(1+\left|X_{s}^{t, x}\right|^{\gamma}\right)^{\frac{p_{0}}{p_{0}-1}}\right]+\mathbf{E}\left[\left(\zeta_{T}\left(C\left(1+\left|\varphi_{n}\left(X_{s}^{t, x}\right)\right|\right) \operatorname{sign}\left(\tilde{Z}_{s}^{1 n}\right)\right)\right)^{p_{0}}\right]\right\} .
\end{aligned}
$$

Finally estimates (4.8) and (3.2) yield that,

$$
\left|\bar{\varpi}^{1 n}(t, x)\right| \leq C\left(1+|x|^{\lambda}\right),
$$


where $\lambda=\frac{\gamma p_{0}}{p_{0}-1}>2$. Next taking into account point (b) and using comparison of solutions of BSDEs ([8], pp.23) we obtain for any $s \in[t, T]$,

$$
\bar{W}_{s}^{1 n}=\bar{\varpi}^{1 n}\left(s, X_{s}^{t, x}\right) \geq W_{s}^{1 n ;(t, x)}=\varpi^{1 n}\left(s, X_{s}^{t, x}\right), \forall s \in[t, T],
$$

and then, choosing $s=t$, we get that $\varpi^{1 n}(t, x) \leq C\left(1+|x|^{\lambda}\right),(t, x) \in[0, T] \times \mathbf{R}^{m}$. But in a similar way one can show that for any $(t, x) \in[0, T] \times \mathbf{R}^{m}$,

$\varpi^{1 n}(t, x) \geq-C\left(1+|x|^{\lambda}\right)$. Therefore $\varpi^{1 n}$ is of polynomial growth w.r.t. $(t, x)$ uniformly in $n$, i.e., it satisfies $(4.5)$.

Step 3: There exists a constant $C$ independent of $n$ and $t, x$ such that for any $t \leq T$, for $i=1,2$,

$$
\mathbf{E}\left[\int_{t}^{T}\left|Z_{s}^{i n ;(t, x)}\right|^{2} d s\right] \leq C .
$$

Actually, we obtain from estimates (4.5) and (3.3) that for any $\alpha \geq 1, i=1,2$,

$$
\mathbf{E}\left[\sup _{t \leq s \leq T}\left|W_{s}^{i n ;(t, x)}\right|^{\alpha}\right] \leq C .
$$

Going back to equation (4.3) and making use of Itô's formula with $\left(W_{s}^{1 n ;(t, x)}\right)^{2}$, we obtain, in a standard way, the result (4.9). The proof is omitted for conciseness.

Step 4: There exists a subsequence of $\left(\left(W_{s}^{i n ;\left(0, x_{0}\right)}, Z_{s}^{i n ;\left(0, x_{0}\right)}\right)_{s \in[0, T]}\right)_{n \geq 1}, i=1,2$, which converges respectively to $\left(W_{s}^{i}, Z_{s}^{i}\right)_{0 \leq s \leq T}, i=1,2$, solution of the BSDE (4.2).

Actually for $i=1,2$ and $n \geq 1$, by (4.4) we know that

$$
W_{s}^{i n ;\left(0, x_{0}\right)}=\varpi^{i n}\left(s, X_{s}^{0, x_{0}}\right), s \leq T
$$

where the deterministic functions $\varpi^{i n}$ verifies:

$$
\forall(t, x) \in[0, T] \times \mathbf{R}^{m}, \varpi^{i n}(t, x)=\mathbf{E}\left[g^{i}\left(X_{T}^{t, x}\right)+\int_{t}^{T} F^{i n}\left(s, X_{s}^{t, x}\right) d s\right] .
$$

Let us now fix $q \in(1,2)$. Taking account of point $(b)$, we have:

$$
\begin{aligned}
\mathbf{E}\left[\int_{0}^{T} \mid F^{i n}\right. & \left.\left.\left(s, X_{s}^{0, x_{0}}\right)\right|^{q} d s\right]=\int_{[0, T] \times \mathbf{R}^{m}}\left|F^{i n}(s, y)\right|^{q} \mu\left(0, x_{0} ; s, d y\right) d s \\
& \leq C \mathbf{E}\left[\int_{0}^{T}\left|Z_{s}^{i n ;\left(0, x_{0}\right)}\right|^{q}\left(1+\left|X_{s}^{0, x_{0}}\right|^{q}\right)+\left(1+\left|X_{s}^{0, x_{0}}\right|^{\gamma q}\right) d s\right] .
\end{aligned}
$$

By Hölder and Young's inequalities, one can show that,

$$
\begin{aligned}
& \mathbf{E}\left[\int_{0}^{T}\left|F^{i n}\left(s, X_{s}^{0, x_{0}}\right)\right|^{q} d s\right] \\
& \leq C \mathbf{E}\left[\left(\int_{0}^{T}\left|Z_{s}^{i n ;\left(0, x_{0}\right)}\right|^{2} d s\right)^{\frac{q}{2}}\left(\int_{0}^{T}\left(1+\left|X_{s}^{0, x_{0}}\right|\right)^{\frac{2 q}{2-q}} d s\right)^{\frac{2-q}{2}}\right] \\
&+C \mathbf{E}\left[\int_{0}^{T}\left(1+\left|X_{s}^{0, x_{0}}\right|^{\gamma q}\right) d s\right] \\
& \leq\left.C\left\{\mathbf{E}\left[\int_{0}^{T}\left|Z_{s}^{i n ;\left(0, x_{0}\right)}\right|^{2} d s\right]+\mathbf{E}\left[1+\sup _{0 \leq s \leq T}\left|X_{s}^{0, x_{0}}\right|^{\theta}\right]\right]\right\}
\end{aligned}
$$


for constant $\theta=(\gamma q) \vee \frac{2 q}{2-q}$ which is greater than 2 with $1<q<2$ and $\gamma>1$. Taking now into account the estimates (4.9) and (3.3) we deduce that,

$$
\mathbf{E}\left[\int_{0}^{T}\left|F^{i n}\left(s, X_{s}^{0, x_{0}}\right)\right|{ }^{q} d s\right]=\int_{[0, T] \times \mathbf{R}^{m}}\left|F^{i n}(s, y)\right|^{q} \mu\left(0, x_{0} ; s, d y\right) d s \leq C .
$$

As a result, there exists a subsequence $\left\{n_{k}\right\}$ (for notational simplification, we still denote it by $\{n\})$ and two $\mathcal{B}([0, T]) \otimes \mathcal{B}\left(\mathbf{R}^{m}\right)$-measurable deterministic functions $F^{i}(s, x), i=1,2$, such that,

$$
F^{i n} \rightarrow F^{i} \quad \text { weakly in } \quad L^{q}\left([0, T] \times \mathbf{R}^{m} ; \mu\left(0, x_{0} ; s, d x\right) d s\right) .
$$

Next we aim to prove that $\left(\varpi^{i n}(t, x)\right)_{n \geq 1}$ is a Cauchy sequence for each $(t, x) \in[0, T] \times \mathbf{R}^{m}$, $\mathrm{i}=1,2$.

So let $(t, x)$ be fixed, $\delta>0, k, n$ and $m \geq 1$ be integers. From (4.10), we have,

$$
\begin{aligned}
\left|\varpi^{i n}(t, x)-\varpi^{i m}(t, x)\right|= & \left|\mathbf{E}\left[\int_{t}^{T}\left[F^{i n}\left(s, X_{s}^{t, x}\right)-F^{i m}\left(s, X_{s}^{t, x}\right)\right] d s\right]\right| \\
\leq & \left|\mathbf{E}\left[\int_{t}^{t+\delta}\left[F^{i n}\left(s, X_{s}^{t, x}\right)-F^{i m}\left(s, X_{s}^{t, x}\right)\right] d s\right]\right| \\
& +\left|\mathbf{E}\left[\int_{t+\delta}^{T}\left(F^{i n}\left(s, X_{s}^{t, x}\right)-F^{i m}\left(s, X_{s}^{t, x}\right)\right) .1_{\left\{\left|X_{s}^{t, x}\right| \leq k\right\}} d s\right]\right| \\
& +\left|\mathbf{E}\left[\int_{t+\delta}^{T}\left(F^{i n}\left(s, X_{s}^{t, x}\right)-F^{i m}\left(s, X_{s}^{t, x}\right)\right) .1_{\left\{\left|X_{s}^{t, x}\right|>k\right\}} d s\right]\right| .
\end{aligned}
$$

On the right side, according to (4.11), we have,

$$
\begin{aligned}
\mathbf{E}\left[\int_{t}^{t+\delta}\right. & \left.\left|F^{i n}\left(s, X_{s}^{t, x}\right)-F^{i m}\left(s, X_{s}^{t, x}\right)\right| d s\right] \\
& \leq \delta^{\frac{q-1}{q}}\left\{\mathbf{E}\left[\int_{t}^{T}\left|F^{i n}\left(s, X_{s}^{t, x}\right)-F^{i m}\left(s, X_{s}^{t, x}\right)\right|^{q} d s\right]\right\}^{\frac{1}{q}} \\
& \leq C \delta^{\frac{q-1}{q}} .
\end{aligned}
$$

At the same time, thanks to Corollary 4.1 associated to $L^{\frac{q}{q-1}}$-domination implies that:

$$
\begin{aligned}
\mid \mathbf{E}\left[\int_{t+\delta}^{T}\right. & \left.\left(F^{i n}\left(s, X_{s}^{t, x}\right)-F^{i m}\left(s, X_{s}^{t, x}\right)\right) \cdot 1_{\left\{\left|X_{s}^{t, x}\right| \leq k\right\}} d s\right] \mid \\
& =\left|\int_{\mathbf{R}^{m}} \int_{t+\delta}^{T}\left(F^{i n}(s, \eta)-F^{i m}(s, \eta)\right) \cdot 1_{\{|\eta| \leq k\}} \mu(t, x ; s, d \eta) d s\right| \\
& =\left|\int_{\mathbf{R}^{m}} \int_{t+\delta}^{T}\left(F^{i n}(s, \eta)-F^{i m}(s, \eta)\right) \cdot 1_{\{|\eta| \leq k\}} \phi_{t, x}(s, \eta) \mu\left(0, x_{0} ; s, d \eta\right) d s\right| .
\end{aligned}
$$

Since $\phi_{t, x}(s, \eta) \in L^{\frac{q}{q-1}}\left([t+\delta, T] \times[-k, k]^{m} ; \mu\left(0, x_{0} ; s, d \eta\right) d s\right)$, for $k \geq 1$, it follows from (4.12) that for each $(t, x) \in[0, T] \times \mathbf{R}^{m}$, we have,

$$
\mathbf{E}\left[\int_{t+\delta}^{T}\left(F^{i n}\left(s, X_{s}^{t, x}\right)-F^{i m}\left(s, X_{s}^{t, x}\right)\right) 1_{\left\{\left|X_{s}^{t, x}\right| \leq k\right\}} d s\right] \rightarrow 0 \text { as } n, m \rightarrow \infty .
$$


Finally,

$$
\begin{aligned}
& \left|\mathbf{E}\left[\int_{t+\delta}^{T}\left(F^{i n}\left(s, X_{s}^{t, x}\right)-F^{i m}\left(s, X_{s}^{t, x}\right)\right) \cdot 1_{\left\{\left|X_{s}^{t, x}\right|>k\right\}} d s\right]\right| \\
& \quad \leq C\left\{\mathbf{E}\left[\int_{t+\delta}^{T} 1_{\left\{\left|X_{s}^{t, x}\right|>k\right\}} d s\right]\right\}^{\frac{q-1}{q}}\left\{\mathbf{E}\left[\int_{t+\delta}^{T}\left|F^{i n}\left(s, X_{s}^{t, x}\right)-F^{i m}\left(s, X_{s}^{t, x}\right)\right|^{q} d s\right]\right\}^{\frac{1}{q}} \\
& \quad \leq C k^{-\frac{q-1}{q}}
\end{aligned}
$$

Therefore, $\left(\varpi^{i n}(t, x)\right)_{n \geq 1}$ is a Cauchy sequence for each $(t, x) \in[0, T] \times \mathbf{R}^{m}$ and then there exists a measurable application $\varpi^{i}$ on $[0, T] \times \mathbf{R}^{m}, i=1,2$, such that for each $(t, x) \in[0, T] \times \mathbf{R}^{m}$, $i=1,2$,

$$
\lim _{n \rightarrow \infty} \varpi^{i n}(t, x)=\varpi^{i}(t, x) .
$$

Additionally we obtain from estimate (4.5) that $\varpi^{i}$ is of polynomial growth, i.e.,

$$
\forall(t, x) \in[0, T] \times \mathbf{R}^{m},\left|\varpi^{i}(t, x)\right| \leq C\left(1+|x|^{\lambda}\right) .
$$

Therefore for any $t \geq 0$.

$$
\lim _{n \rightarrow \infty} W_{t}^{i n ;\left(0, x_{0}\right)}(\omega)=\varpi^{i}\left(t, X_{t}^{0, x_{0}}(\omega)\right),\left|W_{t}^{i n ;\left(0, x_{0}\right)}(\omega)\right| \leq C\left(1+\left|X_{t}^{0, x_{0}}(\omega)\right|^{\lambda}\right), \mathbf{P}-\text { a.s. }
$$

By Lebesgue's dominated convergence theorem, $\left(\left(W_{t}^{i n ;\left(0, x_{0}\right)}\right)_{t \leq T}\right)_{n \geq 1}$ converges to $W^{i}=\left(\varpi^{i}\left(t, X_{t}^{0, x_{0}}\right)\right)_{t \leq T}$ in $\mathcal{H}_{T}^{\kappa}(\mathbf{R})$ for any $\kappa \geq 1$, that is, for $i=1,2$,

$$
\mathbf{E}\left[\int_{0}^{T}\left|W_{s}^{i n ;\left(0, x_{0}\right)}-W_{s}^{i}\right|^{\kappa} d s\right] \rightarrow 0, \quad \text { as } n \rightarrow \infty .
$$

We next show that $\left(W^{i n ;\left(0, x_{0}\right)}\right)_{n \geq 0}$ is convergent in $\mathcal{S}_{T}^{2}(\mathbf{R}), i=1,2$, as well. But first let us show that for $i=1,2$, the sequence $\left(Z^{i n ;\left(0, x_{0}\right)}=\left(v^{i n}\left(t, X_{t}^{0, x_{0}}\right)\right)_{t \leq T}\right)_{n \geq 1}$ has a limit in $\mathcal{H}_{T}^{2}\left(\mathbf{R}^{m}\right)$. As usual, we only deal with the case $i=1$. For $n, m \geq 1$ and $s \leq T$, using Itô's formula with $\left(W_{s}^{1 n}-W_{s}^{1 m}\right)^{2}$ (we omit the subscript $\left(0, x_{0}\right)$ for convenience) and considering point $(b)$, we get,

$$
\begin{aligned}
\left|W_{s}^{1 n}-W_{s}^{1 m}\right|^{2}+\int_{s}^{T}\left|Z_{r}^{1 n}-Z_{r}^{1 m}\right|^{2} d r & \\
= & 2 \int_{s}^{T}\left(W_{r}^{1 n}-W_{r}^{1 m}\right)\left(H_{1}^{n}\left(r, X_{r}^{0, x_{0}}, Z_{r}^{1 n}, Z_{r}^{2 n}\right)-H_{1}^{m}\left(r, X_{r}^{0, x_{0}}, Z_{r}^{1 m}, Z_{r}^{2 m}\right)\right) d r \\
& -2 \int_{s}^{T}\left(W_{r}^{1 n}-W_{r}^{1 m}\right)\left(Z_{r}^{1 n}-Z_{r}^{1 m}\right) d B_{r} \\
\leq & C \int_{s}^{T}\left|W_{r}^{1 n}-W_{r}^{1 m}\right|\left(\left(\left|Z_{r}^{1 n}\right|+\left|Z_{r}^{1 m}\right|\right)\left(1+\left|X_{r}^{0, x_{0}}\right|\right)+\left(1+\left|X_{r}^{0, x_{0}}\right|^{\gamma}\right)\right) d r \\
& -2 \int_{s}^{T}\left(W_{r}^{1 n}-W_{r}^{1 m}\right)\left(Z_{r}^{1 n}-Z_{r}^{1 m}\right) d B_{r} .
\end{aligned}
$$

But for any $x, y, z \in \mathbf{R},|x y z| \leq \frac{1}{p}|x|^{p}+\frac{1}{q}|y|^{q}+\frac{1}{r}|z|^{r}$ with $\frac{1}{p}+\frac{1}{q}+\frac{1}{r}=1$. Then for any $\epsilon>0$ we have,

$$
\begin{aligned}
\mid & W_{s}^{1 n}-\left.W_{s}^{1 m}\right|^{2}+\int_{s}^{T}\left|Z_{r}^{1 n}-Z_{r}^{1 m}\right|^{2} d r \\
\leq & C\left\{\frac{\epsilon^{2}}{2} \int_{s}^{T}\left(\left|Z_{r}^{1 n}\right|+\left|Z_{r}^{1 m}\right|\right)^{2} d r+\frac{\epsilon^{4}}{4} \int_{s}^{T}\left(1+\left|X_{r}^{0, x_{0}}\right|\right)^{4} d r+\frac{1}{4 \epsilon^{8}} \int_{s}^{T}\left|W_{r}^{1 n}-W_{r}^{1 m}\right|^{4} d r+\right. \\
& \left.\int_{s}^{T}\left|W_{r}^{1 n}-W_{r}^{1 m}\right|\left(1+\left|X_{r}^{0, x_{0}}\right|^{\gamma}\right) d r\right\}-2 \int_{s}^{T}\left(W_{r}^{1 n}-W_{r}^{1 m}\right)\left(Z_{r}^{1 n}-Z_{r}^{1 m}\right) d B_{r} .
\end{aligned}
$$


Taking now $s=0$, expectation on both sides and the limit w.r.t. $n$ and $m$ we deduce that,

$$
\limsup _{n, m \rightarrow \infty} \mathbf{E}\left[\int_{0}^{T}\left|Z_{r}^{1 n}-Z_{r}^{1 m}\right|^{2} d r\right] \leq C\left\{\frac{\epsilon^{2}}{2}+\frac{\epsilon^{4}}{4}\right\}
$$

due to estimates (4.9), (3.3) and the convergence of (4.14). As $\epsilon$ is arbitrary then the sequence $\left(Z^{1 n}\right)_{n \geq 0}$ is convergent in $\mathcal{H}_{T}^{2}\left(\mathbf{R}^{m}\right)$ to a process $Z^{1}$.

Next once more going back to inequality (4.15), taking the supremum and using BDG's inequality we deduce that,

$$
\begin{aligned}
& \mathbf{E}\left[\sup _{0 \leq s \leq T}\left|W_{s}^{1 n}-W_{s}^{1 m}\right|^{2}+\int_{0}^{T}\left|Z_{r}^{1 n}-Z_{r}^{1 m}\right|^{2} d r\right] \\
& \leq C \mathbf{E}\left\{\frac{\epsilon^{2}}{2} \int_{0}^{T}\left(\left|Z_{r}^{1 n}\right|+\left|Z_{r}^{1 m}\right|\right)^{2} d r+\frac{\epsilon^{4}}{4} \int_{0}^{T}\left(1+\left|X_{r}^{0, x_{0}}\right|\right)^{4} d r+\frac{1}{4 \epsilon^{8}} \int_{0}^{T}\left|W_{r}^{1 n}-W_{r}^{1 m}\right|^{4} d r\right. \\
& \left.\quad+\int_{0}^{T}\left|W_{r}^{1 n}-W_{r}^{1 m}\right|\left(1+\left|X_{r}^{0, x_{0}}\right|^{\gamma}\right) d s\right\}+\frac{1}{4} \mathbf{E}\left[\sup _{0 \leq s \leq T}\left|W_{s}^{1 n}-W_{s}^{1 m}\right|^{2}\right] \\
& \quad+4 \mathbf{E}\left[\int_{0}^{T}\left|Z_{r}^{1 n}-Z_{r}^{1 m}\right|^{2} d r\right] .
\end{aligned}
$$

which implies that,

$$
\limsup _{n, m \rightarrow \infty} \mathbf{E}\left[\sup _{0 \leq s \leq T}\left|W_{s}^{1 n}-W_{s}^{1 m}\right|^{2}\right]=0
$$

since $\epsilon$ is arbitrary. Thus the sequence of processes $\left(W^{1 n}\right)_{n \geq 1}$ converges in $\mathcal{S}_{T}^{2}(\mathbf{R})$ to $W^{1}$ which is a continuous process.

Finally note that we can do the same for $i=2$, i.e., we have also the convergence of $\left(Z^{2 n}\right)_{n>0}$ $\left(\operatorname{resp} .\left(W^{2 n}\right)_{n \geq 0}\right)$ in $\mathcal{H}_{T}^{2}\left(\mathbf{R}^{m}\right)\left(\operatorname{resp} . \mathcal{S}_{T}^{2}(\mathbf{R})\right)$ to $Z^{2}\left(\operatorname{resp} .\left(W_{t}^{2}=\varpi^{2}\left(t, X_{t}^{0, x_{0}}\right)\right)_{t \leq T}\right)$.

Step 5: The limit processes $\left(W_{s}^{i}, Z_{s}^{i}\right)_{s \leq T}, i=1,2$, are solutions of BSDE (4.2). Indeed, we need to prove that (for case $i=1$ ),

$$
F^{1}\left(t, X_{t}^{0, x_{0}}\right)=H_{1}\left(t, X_{t}^{0, x_{0}}, Z_{t}^{1},\left(u_{1}^{*}, u_{2}^{*}\right)\left(t, X_{t}^{0, x_{0}}, Z_{t}^{1}, Z_{t}^{2}\right)\right) \quad d t \otimes d \mathbf{P}-\text { a.s. }
$$

For $k \geq 1$, we have,

$$
\begin{aligned}
& \mathbf{E}\left[\int_{0}^{T}\left|H_{1}^{n}\left(s, X_{s}^{0, x_{0}}, Z_{s}^{1 n}, Z_{s}^{2 n}\right)-H_{1}\left(s, X_{s}^{0, x_{0}}, Z_{s}^{1},\left(u_{1}^{*}, u_{2}^{*}\right)\left(s, X_{s}^{0, x_{0}}, Z_{s}^{1}, Z_{s}^{2}\right)\right)\right| d s\right] \\
= & \mathbf{E}\left[\int_{0}^{T} \mid H_{1}^{n}\left(s, X_{s}^{0, x_{0}}, Z_{s}^{1 n}, Z_{s}^{2 n}\right)\right. \\
+ & \mathbf{E}\left[\int_{0}^{T} \mid H_{1}^{n}\left(s, X_{s}^{0, x_{0}}, Z_{s}^{1 n}, Z_{s}^{2 n}\right)\right. \\
& \left.\quad-H_{1}\left(s, X_{s}^{0, x_{0}}, Z_{s}^{1 n},\left(u_{1}^{*}, u_{2}^{*}\right)\left(s, X_{s}^{0, x_{0}}, Z_{s}^{1 n}, Z_{s}^{2 n}\right)\right) \mid \cdot 1_{\left\{\left|Z_{s}^{1 n}\right|+\left|Z_{s}^{2 n}\right|<k\right\}} d s\right] \\
+ & \left.\mathbf{E}\left[\int_{0}^{T} \mid H_{1}\left(s, X_{s}^{0, x_{0}}, Z_{s}^{1 n}\right)\left(u_{1}^{*}, X_{s}^{*}, u_{2}^{*}\right)\left(s, X_{s}^{0, x_{0}}, Z_{s}^{1 n}, Z_{s}^{1 n}, Z_{s}^{2 n}\right)\right) \mid \cdot 1_{\left\{\left|Z_{s}^{1 n}\right|+\left|Z_{s}^{2 n}\right| \geq k\right\}} d s\right] \\
& \left.-H_{1}\left(s, X_{s}^{0, x_{0}}, Z_{s}^{1},\left(u_{1}^{*}, u_{2}^{*}\right)\left(s, X_{s}^{0, x_{0}}, Z_{s}^{1}, Z_{s}^{2}\right)\right) \mid d s\right] .
\end{aligned}
$$


The first term converges to 0 . Indeed, on one hand, for $n \geq 1$, point $(b)$ implies that,

$$
\begin{gathered}
\left|H_{1}^{n}\left(s, X_{s}^{0, x_{0}}, Z_{s}^{1 n}, Z_{s}^{2 n}\right)-H_{1}\left(s, X_{s}^{0, x_{0}}, Z_{s}^{1 n},\left(u_{1}^{*}, u_{2}^{*}\right)\left(s, X_{s}^{0, x_{0}}, Z_{s}^{1 n}, Z_{s}^{2 n}\right)\right)\right| \cdot 1_{\left\{\left|Z_{s}^{1 n}\right|+\left|Z_{s}^{2 n}\right|<k\right\}} \\
\leq C\left(1+\left|X_{s}^{0, x_{0}}\right|\right) k+C\left(1+\left|X_{s}^{0, x_{0}}\right|^{\gamma}\right) .
\end{gathered}
$$

On the other hand, considering point $(d)$, we obtain that,

$$
\begin{gathered}
\left|H_{1}^{n}\left(s, X_{s}^{0, x_{0}}, Z_{s}^{1 n}, Z_{s}^{2 n}\right)-H_{1}\left(s, X_{s}^{0, x_{0}}, Z_{s}^{1 n},\left(u_{1}^{*}, u_{2}^{*}\right)\left(s, X_{s}^{0, x_{0}}, Z_{s}^{1 n}, Z_{s}^{2 n}\right)\right)\right| \cdot 1_{\left\{\left|Z_{s}^{1 n}\right|+\left|Z_{s}^{2 n}\right|<k\right\}} \\
\leq \sup _{\left\{\left(z_{s}^{1}, z_{s}^{2}\right),\left|z_{s}^{1}\right|+\left|z_{s}^{2}\right| \leq k\right\}} \mid H_{1}^{n}\left(s, X_{s}^{0, x_{0}}, z_{s}^{1}, z_{s}^{2}\right) \\
\quad-H_{1}\left(s, X_{s}^{0, x_{0}}, z_{s}^{1},\left(u_{1}^{*}, u_{2}^{*}\right)\left(s, X_{s}^{0, x_{0}}, z_{s}^{1}, z_{s}^{2}\right)\right) \mid \rightarrow 0 \text { as } n \rightarrow \infty .
\end{gathered}
$$

Then thanks to Lebesgue's dominated convergence theorem, the first term in (4.17) converges to 0 in $\mathcal{H}_{T}^{1}(\mathbf{R})$.

The second term is bounded by $\frac{C}{k^{2(q-1) / q}}$ with $q \in(1,2)$. Actually, from point $(b)$ and Markov's inequality, for $1<q<2$ we have,

$$
\begin{aligned}
& \mathbf{E}\left[\int_{0}^{T} \mid H_{1}^{n}\left(s, X_{s}^{0, x_{0}}, Z_{s}^{1 n}, Z_{s}^{2 n}\right)\right. \\
& \left.\quad-H_{1}\left(s, X_{s}^{0, x_{0}}, Z_{s}^{1 n},\left(u_{1}^{*}, u_{2}^{*}\right)\left(s, X_{s}^{0, x_{0}}, Z_{s}^{1 n}, Z_{s}^{2 n}\right)\right) \mid \cdot 1_{\left\{\left|Z_{s}^{1 n}\right|+\left|Z_{s}^{2 n}\right| \geq k\right\}} d s\right] \\
\leq & C\left\{\mathbf{E}\left[\int_{0}^{T}\left(1+\left|X_{s}^{0, x_{0}}\right|\right)^{q}\left|Z_{s}^{1 n}\right|^{q}+\left(1+\left|X_{s}^{0, x_{0}}\right|^{\gamma}\right)^{q} d s\right]\right\}^{\frac{1}{q}}\left\{\mathbf{E}\left[\int_{0}^{T} 1_{\left\{\left|Z_{s}^{1 n}\right|+\left|Z_{s}^{2 n}\right| \geq k\right\}} d s\right]\right\}^{\frac{q-1}{q}} \\
\leq & C\left\{\mathbf{E}\left[\int_{0}^{T} 1_{\left\{\left|Z_{s}^{1 n}\right|+\left|Z_{s}^{2 n}\right| \geq k\right\}} d s\right]\right\}^{\frac{q-1}{q}} \\
\leq & \frac{C}{k^{\frac{2(q-1)}{q}} .}
\end{aligned}
$$

The second inequality is obtained by Young's inequality and estimates (4.9) and (3.3).

The third term in (4.17) also converges to 0, at least for a subsequence. Actually, since the sequence $\left(Z^{1 n}\right)_{n \geq 1}$ converges to $Z^{1}$ in $\mathcal{H}_{T}^{2}\left(\mathbf{R}^{m}\right)$, there exists a subsequence $\left(Z^{1 n_{k}}\right)_{k \geq 0}$ which converges to $Z^{1}, d t \otimes d \mathbf{P}$-a.e and such that $\sup _{k \geq 0}\left|Z_{t}^{1 n_{k}}(\omega)\right|$ belongs to $\mathcal{H}_{T}^{2}(\mathbf{R})$. Therefore, taking the continuity Assumption (A3)-(ii) of $H_{1}\left(t, x, p,\left(u_{1}^{*}, u_{2}^{*}\right)(t, x, p, q)\right)$ w.r.t $(p, q)$, we obtain that,

$$
\begin{aligned}
H_{1}\left(s, X_{s}^{0, x_{0}}, Z_{s}^{1 n_{k}},\left(u_{1}^{*}, u_{2}^{*}\right)\left(s, X_{s}^{0, x_{0}}, Z_{s}^{1 n_{k}}, Z_{s}^{2 n_{k}}\right)\right) & \\
\quad \longrightarrow k \rightarrow \infty & H_{1}\left(s, X_{s}^{0, x_{0}}, Z_{s}^{1},\left(u_{1}^{*}, u_{2}^{*}\right)\left(s, X_{s}^{0, x_{0}}, Z_{s}^{1}, Z_{s}^{2}\right)\right) d t \otimes d \mathbf{P}-\text { a.e. }
\end{aligned}
$$

and the process

$$
\sup _{k \geq 0}\left|H_{1}\left(s, X_{s}^{0, x_{0}}, Z_{s}^{1 n_{k}},\left(u_{1}^{*}, u_{2}^{*}\right)\left(s, X_{s}^{0, x_{0}}, Z_{s}^{1 n_{k}}, Z_{s}^{2 n_{k}}\right)\right)\right| \in \mathcal{H}_{T}^{q}(\mathbf{R}) .
$$

Then once more by the dominated convergence theorem, we obtain,

$$
\begin{aligned}
& H_{1}\left(s, X_{s}^{0, x_{0}}, Z_{s}^{1 n_{k}},\left(u_{1}^{*}, u_{2}^{*}\right)\left(s, X_{s}^{0, x_{0}}, Z_{s}^{1 n_{k}}, Z_{s}^{2 n_{k}}\right)\right) \\
& \quad \longrightarrow{ }_{k \rightarrow \infty} H_{1}\left(s, X_{s}^{0, x_{0}}, Z_{s}^{1},\left(u_{1}^{*}, u_{2}^{*}\right)\left(s, X_{s}^{0, x_{0}}, Z_{s}^{1}, Z_{s}^{2}\right)\right) \text { in } \mathcal{H}_{T}^{q}(\mathbf{R}),
\end{aligned}
$$


which yields to the convergence of the third term in (4.17).

It follows that the sequence $\left(\left(H_{1}^{n}\left(s, X_{s}^{0, x_{0}}, Z_{s}^{1 n}, Z_{s}^{2 n}\right)_{s<T}\right)_{n>1}\right.$ converges to $\left(H_{1}\left(s, X_{s}^{0, x_{0}}, Z_{s}^{1},\left(u_{1}^{*}, u_{2}^{*}\right)\left(s, X_{s}^{0, x_{0}}, Z_{s}^{1}, Z_{s}^{2}\right)\right)\right)_{s \leq T}$ in $L^{1}([0, T] \times \Omega, d t \otimes d \mathbf{P})$ and then

$$
F^{1}\left(s, X_{s}^{0, x_{0}}\right)=H_{1}\left(s, X_{s}^{0, x_{0}}, Z_{s}^{1},\left(u_{1}^{*}, u_{2}^{*}\right)\left(s, X_{s}^{0, x_{0}}, Z_{s}^{1}, Z_{s}^{2}\right)\right), d t \otimes d \mathbf{P}-\text { a.e. }
$$

In the same way we have,

$$
F^{2}\left(s, X_{s}^{0, x_{0}}\right)=H_{2}\left(s, X_{s}^{0, x_{0}}, Z_{s}^{2},\left(u_{1}^{*}, u_{2}^{*}\right)\left(s, X_{s}^{0, x_{0}}, Z_{s}^{1}, Z_{s}^{2}\right)\right), d t \otimes d \mathbf{P}-a . s .
$$

Thus the processes $\left(W^{i}, Z^{i}\right), i=1,2$, is solution of the backward equation (4.2). Finally taking into account of estimate (4.13) and the fact that $Z^{i}, i=1,2$, belong to $\mathcal{H}_{T}^{2}\left(\mathbf{R}^{m}\right)$ complete the proof.

As a result of Theorems 3.1 and 4.1 we obtain the main result of this paper.

Theorem 4.2. Assume that (A1), (A2) and (A3) are in force. Then the nonzero-sum differential game defined by (2.4) and (2.5) has a Nash equilibrium point.

Example: The linear quadratic case.

Let us take $m=1$ and for $t \in[0, T], X_{t}^{0, x_{0}}=x_{0}+B_{t}$. Let $f(t, x, u, v)=a x+b u+c v$ and for $i=1,2, h_{i}(t, x, u, v)=\theta_{i} x^{p_{i}}+\gamma_{i} u^{2}+\rho_{i} v^{2}, U=[-1,1]$ and $V=[0,1]$ where $a, b, c, p_{i}$, $\rho_{i} \gamma_{i}$ are real constants such that $p_{i} \geq 0, \gamma_{1}>0$ and $\rho_{2}>0$. Finally let $g^{i}, i=1,2$, be two Borel measurable functions with polynomial growth. The Hamiltonian functions $H_{i}$ of the nonzero-sum differential game associated with $X^{0, x_{0}}, f, h_{i}, g_{i}, i=1,2$, and $U, V$ are:

$$
H_{i}\left(t, x, z_{i}, u, v\right)=z_{i} f(t, x, u, v)+h_{i}(t, x, u, v), i=1,2 .
$$

Next for $\eta \in \mathbf{R}$ let $\psi$ and $\phi$ be functions from $\mathbf{R}$ to $\mathbf{R}$ defined by:

$$
\psi(\eta):=-1_{[\eta<-1]}+\eta 1_{[-1 \leq \eta \leq 1]}+1_{[\eta>1]} \text { and } \phi(\eta):=1 \wedge \eta^{+} .
$$

Thus the functions $u^{*}\left(t, x, z_{1}, z_{2}\right):=\psi\left(-\frac{b z_{1}}{2 \gamma_{1}}\right)$ and $v^{*}\left(t, x, z_{1}, z_{2}\right):=\phi\left(-\frac{c z_{2}}{2 \rho_{2}}\right)$ (which are continuous in $\left.\left(z_{1}, z_{2}\right)\right)$ verify the generalized Isaacs condition (A3). Therefore, according to Theorem 4.2, this game has a Nash equilibrium point $\left(u^{*}\left(t, X_{t}^{0, x_{0}}, Z_{1}(t), Z_{2}(t)\right), v^{*}\left(t, X_{t}^{0, x_{0}}, Z_{1}(t), Z_{2}(t)\right)\right)_{t \leq T}$.

Remark 4.1. For sake of simplicity we have dealt with the case of two players. However the method still work if we have more than two players. We just need a minor adaptation of the generalized Isaacs condition of (A3). Actually assume there are $N$ players $P_{1}, \ldots, P_{N}(N \geq 3)$ and for $i=1, \ldots, N$, let $U_{i}$ be the compact set where the controls of player $P_{i}$ take their values. Let $\left(H_{i}\right)_{i=1, \ldots, N}$ be the Hamiltonian functions of the nonzero-sum differential game associated with $f\left(t, x, u_{1}, \ldots, u_{N}\right), h_{i}\left(t, x, u_{1}, \ldots, u_{N}\right)$ and $g^{i}$, i.e.,

$$
H_{i}\left(t, x, z_{i}, u_{1}, \ldots, u_{N}\right):=z_{i} \sigma^{-1}(t, x) . f\left(t, x, u_{1}, \ldots, u_{N}\right)+h_{i}\left(t, x, u_{1}, \ldots, u_{N}\right) .
$$

We assume that, uniformly w.r.t. $x, f$ is of linear growth and $h_{i}, g^{i}, i=1, \ldots, N$, are of polynomial growth. Next assume that generalized Isaacs condition, which reads as below, is satisfied: 
(i) There exist $N$ Borel functions $u_{1}^{*}, \ldots, u_{N}^{*}$ defined on $[0, T] \times \mathbf{R}^{(N+1) m}$, valued respectively in $U_{1}, \ldots, U_{N}$, such that for any $i=1, \ldots, N,\left(t, x, z_{1}, \ldots, z_{N}\right) \in[0, T] \times \mathbf{R}^{(N+1) m}$, we have:

$$
\begin{aligned}
& H_{i}\left(t, x, z_{i},\left(u_{1}^{*}, \ldots, u_{N}^{*}\right)\left(t, x, z_{1}, \ldots, z_{N}\right)\right) \\
& \leq H_{i}\left(t, x, z_{i},\left(u_{1}^{*}, \ldots, u_{i-1}^{*}\right)\left(t, x, z_{1}, \ldots, z_{N}\right), u_{i},\left(u_{i+1}^{*}, \ldots, u_{N}^{*}\right)\left(t, x, z_{1}, \ldots, z_{N}\right)\right), \forall u_{i} \in U_{i} ;
\end{aligned}
$$

(ii) For any fixed $(t, x)$ the mapping

$$
\left(z_{1}, \ldots, z_{N}\right) \longmapsto H_{i}\left(t, x, z_{i},\left(u_{1}^{*}, \ldots, u_{N}^{*}\right)\left(t, x, z_{1}, \ldots, z_{N}\right)\right)
$$

is continuous.

Then, if $\sigma(t, x)$ verifies the Assumption (A1), the differential game associated with the drift $f\left(t, x, u_{1}, \ldots, u_{N}\right)$, the instantaneous payoffs $h_{i}\left(t, x, u_{1}, \ldots, u_{N}\right)$ and the terminal payoffs $g^{i}(x)$, $i=1, \ldots, N$, has a Nash equilibrium point.

Extension: In this study the main points we required are:

(i) the existence of $p>1$ such that for any pair $(u, v) \in \mathcal{M}, \mathbf{E}\left[\left(\zeta_{T}(u, v)\right)^{p}\right]<\infty$ where $\zeta_{T}(u, v)$ is defined as in $(2.2)$;

(ii) the $L^{q}$-domination property or its adaptation ;

(iii) the generalized Isaacs condition.

As far as those points are in force, one can expect that the NZSDG has a NEP and, e.g., one can let drop Hörmander's condition (2.1) on $\sigma$. Actually let us consider the following example where the matrix $\sigma$ is non longer bounded and does satisfy (2.1). Assume that $m=1$ and for $(t, x) \in[0, T] \times \mathbf{R}, X^{t, x}$ is solution of:

$$
d X_{s}^{t, x}=X_{s}^{t, x} d B_{s}, s \in[t, T] \text { and } X_{s}^{t, x}=x>0 \text { for } s \in[0, t] .
$$

Note that

$$
\forall s \leq T, X_{s}^{t, x}>0
$$

Next let

$$
f(s, x, u, v):=x\left(u_{s}+v_{s}\right), s \leq T, U=[-1,1], V=[0,1]
$$

and assume that $h_{1}$ and $h_{2}$ are as in the previous example. Therefore the generalized Isaacs condition is satisfied with

$$
u^{*}\left(t, x, z_{1}, z_{2}\right):=\psi\left(-\frac{z_{1}}{2 \gamma_{1}}\right) \text { and } v^{*}\left(t, x, z_{1}, z_{2}\right):=\phi\left(-\frac{z_{2}}{2 \rho_{2}}\right),
$$

where $\psi$ and $\phi$ are the functions defined above.

Obviously for any $(u, v) \in \mathcal{M}, \zeta_{T}\left(\left(u_{s}+v_{s}\right)\right)$ belongs to $L^{p}(\mathbf{P})$ for any $p>1$ since $U$ and $V$ are bounded sets. Next for any $s \in[t, T]$, we have:

$$
X_{s}^{t, x}=x \exp \left\{B_{s}-B_{t}-\frac{1}{2}(s-t)\right\} .
$$

Thus for $s \in] t, T]$, the law of $X_{s}^{t, x}$ has a density $p(t, x ; s, y)$ given by

$$
p(t, x ; s, y):=\frac{1}{\sqrt{2 \pi(s-t)}} \exp \left\{-\frac{1}{2(s-t)}\left[\ln \left(\frac{y}{x}\right)+\frac{1}{2}(s-t)\right]^{2}\right\} 1_{[y>0]}, y \in \mathbf{R} .
$$

So let $x_{0}>0, \delta>0(\delta+t<T)$ and $\Phi$ defined by:

$$
\Phi(s, y):=\frac{p(t, x ; s, y)}{p\left(0, x_{0} ; s, y\right)},(s, y) \in[t+\delta, T] \times \mathbf{R} .
$$


Therefore for any $\kappa>0, \Phi$ belongs to $L^{q}\left([t+\delta, T] \times\left[\frac{1}{\kappa}, \kappa\right], p\left(0, x_{0} ; s, y\right) d s d y\right)$ which is the adaptation of property (ii) of Definition 4.1 on $L^{q}$-domination, in taking into account (4.18). Now as the following estimate holds true:

$$
\mathbf{E}\left[\int_{t+\delta}^{T}\left\{1_{\left[X_{s}^{t, x}<\kappa^{-1}\right]}+1_{\left[X_{s}^{t, x}>\kappa\right]}\right\} d s\right] \leq \varrho(\kappa)
$$

where the function $\varrho$ is such that $\varrho(\kappa) \rightarrow 0$ as $\kappa \rightarrow \infty$. Then one can conclude that the NZSDG defined with those specific data $\sigma, U, V, f, g_{i}, h_{i}, i=1,2$, has a Nash equilibrium point. The proof can be established in the same way as we did in the previous sections.

\section{Acknowledgement}

We are grateful to the two anonymous referees for useful comments and suggestions.

\section{References}

[1] D.G. Aronson, Bounds for the fundamental solution of a parabolic equation, Bulletin of the American Mathematical society. 73.6 (1967), pp. 890-896.

[2] A. Bensoussan and J.L. Lions, Application of variational inequalities in stochastic control, Studies in Mathematics and its Applications, vol. 12. North-Holland Publishing Co., Amsterdam-New York, 1982.

[3] R. Buckdahn and J. Li, Stochastic differential games and viscosity solutions for HamiltonJacobi-Bellman-Isaacs equations, SIAM journal on control and optimization. 47.1(2008), pp. $444-475$.

[4] R. Buckdahn, P. Cardaliaguet and C. Rainer, Nash equilibrium payoffs for nonzero-sum stochastic differential games, SIAM journal on control and optimization. 43.2(2004), pp. 624-642.

[5] M.H.A. Davis and R.J. Elliott, Optimal play in a stochastic differential game, SIAM journal on control and optimization. 19.4 (1981), pp. 543-554.

[6] R. J. Elliott, The existence of value in stochastic differential games, SIAM journal on control and optimization. 14.1 (1976), pp. 85-94.

[7] N. El-Karoui and S. Hamadène BSDEs and risk-sensitive control, zero-sum and nonzerosum game problems of stochastic functional differential equations, Stochastic Processes and their Applications, 107.1 (2003), pp.145-169.

[8] N. El-Karoui, S. Peng and M.C. Quenez, Backward stochastic differential equations in finance, Mathematical finance, 7.1(1997), pp. 1-71.

[9] W. H. Fleming and P. E. Souganidis On the existence of value functions of two-player, zero-sum stochastic differential games, Indiana Univ. Math. J. 38.2(1989), pp. 293-314.

[10] A. Friedman, Differential games, Wiley, New York, 1971.

[11] A. Friedman, Stochastic differential games, J. Differential Equations, 11.1(1972), pp. 79-108. 
[12] I.V. Girsanov, On transforming a certain class of stochastic processes by absolutely continuous substitution of measures, Theory of Probability and its Applications, 5(1960), pp. 285.

[13] S. Hamadène and J.-P. Lepeltier, Points d'équilibre dans les jeux stochastiques de somme non nulle, Comptes rendus de l' Académie des sciences, tome 318, série 1, fev.94(1994), pp.251-256.

[14] S. Hamadène and J.-P. Lepeltier, Zero-sum stochastic differential games and backward equations, Systems \& Control Letters, 24.4(1995a), pp. 259-263.

[15] S. Hamadène and J.-P. Lepeltier, Backward equations, stochastic control and zero-sum stochastic differential games, Stochastics: An International Journal of Probability and Stochastic Processes, 54(1995b), pp. 221-231.

[16] S. Hamadène, J.-P. Lepeltier and S. Peng,BSDEs with continuous coefficients and stochastic differential games, Pitman Research Notes in Mathematics Series, (1997), pp. 115-128.

[17] S. Hamadène, Nonzero sum linear-quadratic stochastic differential games and backwardforward equations, Stochastic Analysis and Applications. 17.1(1999), pp. 117-130.

[18] S. Hamadène, Backward-forward SDEs and stochastic differential games, Stochastic Processes and their Applications, 77.1(1998), pp.1-15.

[19] U.G. Haussmann, A stochastic maximum principle for optimal control of diffusions, John Wiley \& Sons, Inc. 1986.

[20] R. Isaacs, Differential Games, Wiley, New York, 1965.

[21] I. Karatzas and S.E. Shreve, Brownian Motion and Stochastic Calculus - 2nd ed., Springer Verlag, 1991.

[22] J.P. Lepeltier, Z. Wu and Z. Yu, Nash equilibrium point for one kind of stochastic nonzerosum game problem and BSDEs, Comptes Rendus Mathematique, 347.15 (2009), pp. 959964.

[23] Q. Lin, A BSDE approach to Nash equilibrium payoffs for stochastic differential games with nonlinear cost functionals, Stochastic Processes and their Applications,122.1(2012), pp. 357-385.

[24] P. Mannucci, Nonzero-sum stochastic differential games with discontinuous feedback, SIAM journal on control and optimization, 43.4(2004), pp. 1222-1233.

[25] E. Pardoux and S. Peng, Adapted solution of a backward stochastic differential equation, Systems \& Control Letters, 14.1(1990), pp. 55-61.

[26] T. Pham and J. Zhang, Two person zero-sum game in weak formulation and path dependent Bellman-Isaacs equation, (2012), preprint arXiv:1209.6605v1.

[27] P. Protter, Stochastic Integration and Differential Equations, 2nd ed., Springer-Verlag, 2004.

[28] C. Rainer, Two different approaches to nonzero-sum stochastic differential games, Applied Mathematics and Optimization. 56.1(2007), pp. 131-144. 
[29] M. Sirbu, Stochastic Perrons method and elementary strategies for zero-sum dierential games, (2013), preprint arXiv:1305.5083v1. 